\title{
Stability Analysis for Neutral Delay Markovian Jump Systems with Nonlinear Perturbations and Partially Unknown Transition Rates
}

\author{
Xinghua Liu and Hongsheng Xi \\ Department of Auto, School of Information Science and Technology, University of Science and Technology of China, \\ Anhui 230027, China \\ Correspondence should be addressed to Xinghua Liu; salxh@mail.ustc.edu.cn
}

Received 1 May 2013; Accepted 13 June 2013

Academic Editor: Shao-Ming Fei

Copyright (C) 2013 X. Liu and H. Xi. This is an open access article distributed under the Creative Commons Attribution License, which permits unrestricted use, distribution, and reproduction in any medium, provided the original work is properly cited.

\begin{abstract}
The problem of exponential stability for the uncertain neutral Markovian jump systems with interval time-varying delays and nonlinear perturbations is investigated in this paper. This study starts from the corresponding nominal systems with known and partially unknown transition rates, respectively. By constructing a novel augmented Lyapunov functional which contains tripleintegral terms and fully utilizes the bound of the delay, the delay-range-dependent and rate-dependent exponential stability criteria are developed by the Lyapunov theory, reciprocally convex lemma, and free weighting matrices. Then, the results about nominal systems are extended to the uncertain case. Finally, numerical examples are given to demonstrate the effectiveness of the proposed methods.
\end{abstract}

\section{Introduction}

Neutral time-delay systems have been the focus of the research community, which are often encountered in such practical situations as distributed networks, population ecology, processes including steam or heat exchanges [1], and robots in contact with rigid environments [2], and so forth. The existence of time delay may cause the instability of the systems, thus making the stability analysis of timedelay systems an interesting topic. Existing results can be roughly classified into two categories, delay-independent criteria and delay-dependent criteria, where the latter is generally regarded as less conservative. In addition, it should be pointed out that the stability of neutral time-delay systems is more difficult to tackle since the derivative of the delayed state is involved. The situation is similar as singular systems $[3,4]$, whose stability problem is more complicated than that for regular systems because more factors need to be considered. In the past decades, considerable attention has been devoted to the robust delay-independent stability and delay-dependent stability of linear neutral systems, which are mainly obtained based on the Lyapunov-Krasovskii (L-K) method [5-8]. Furthermore, when nonlinear perturbations or parameter uncertainties appear in neutral systems, some results on stability analysis have been also presented [914]. Various techniques have been proposed in these papers, for example, model transformation techniques, the improved bounding techniques, and matrix decomposition approaches. In particular, He et al. [14] propose a new method for dealing with time-delay systems, which employs free weighting matrices to express the relationships between the terms in the Newton-Leibniz formula and has brought novel results. However, many complex systems with uncertainties and neutral types as well as time-varying or state-dependent delays are still inviting further investigation.

It is noted that many practical dynamics such as solar thermal central receivers, robotic manipulator systems, aircraft control systems, and economic systems, experience abrupt changes in their structures, caused by phenomena such as component failures or repairs, changes in subsystem interconnections, and sudden environmental changes. This class of systems can be described as Markovian jump systems (MJSs) where the abrupt variation in the structures and parameters can be naturally represented by the jumps in MJSs. Since its first introduction by Krasovskii and Lidskii in 1961, MJSs have received much attention, 
and considerable progress has been made; see, for example, [15-22] and references therein for more details. In view of these results, although related research has made good achievement, we have to admit that the transition probabilities in the jumping process determine the system behavior to a large extent. However, the likelihood of obtaining such available knowledge is actually questionable, and the cost is probably expensive. Thus, it is significant and necessary, from control perspectives, to further study more general jump systems with partly unknown transition probabilities. Recently, many results on the Markovian jump systems with partly unknown transition probabilities are obtained [23-27]. Most of these improved results just require some free matrices or the knowledge of the known elements in transition rate matrix, such as the bounds or structures of uncertainties, and some else of the unknown elements do not need to be considered. It is a great progress on the analysis of Markovian jump systems. However, few of these results are concerned with neutral delay systems. It is urgent and significant to consider the problem of delay-dependent exponential stability for Markovian jumping neutral systems with partially unknown transition rates. Besides, to the best of the authors' knowledge, the neutral Markovian jump systems have not been fully investigated, and it is very challenging, especially when nonlinear perturbations exist. These facts thus motivate our study.

In this paper, the exponential stability problem of neutral Markovian jump systems with mixed interval time-varying delay, nonlinear perturbations, and partially unknown transition rates is investigated. A new augmented Lyapunov functional containing triple-integral terms is constructed by dividing the delay interval into two subintervals, and then the delay-range-dependent and rate-dependent exponential stability criteria are obtained by reciprocally convex lemma and free weighting matrices. Moreover, in contrast with the recent research on uncertain transition rates, our proposed concept of the partly unknown transition rates does not require any knowledge of the unknown elements, such as the bounds or structures of uncertainties. On the basis of the obtained results about nominal systems, we further extend the criteria to the uncertain case. All the obtained results are presented in terms of LMIs that can be solved numerically.

The main contributions of this paper can be summarized as follows: (1) the bound of the delay is fully utilized in this paper; that is, improved bounding technique is used to reduce the conservativeness. (2) The constructed Lyapunov functional contains some triple-integral terms which is very effective in the reduction of conservativeness and has not appeared in the context of neutral Markovian jump systems with nonlinear perturbations before. (3) The reciprocally convex lemma is used to derive the delay-range-dependent stability conditions, which can reduce the conservativeness of the investigated systems. (4) The proposed results are applicable to the uncertain transition rates and expressed in a new representation, which are proved to be less conservative than some existing ones.

The remainder of the paper is organized as follows. Section 2 presents the problem and preliminaries. Section 3 gives the main results, which are then verified by numerical examples in Section 4. Section 5 concludes the paper.

Notation. The following notations are used throughout the paper. $\mathbb{R}^{n}$ denotes the $n$ dimensional Euclidean space, and $\mathbb{R}^{m \times n}$ is the set of all $m \times n$ matrices. $X<Y(X>Y)$, where $X$ and $Y$ are both symmetric matrices, means that $X-Y$ is negative (positive) definite. $I$ is the identity matrix with proper dimensions. For a symmetric block matrix, we use $*$ to denote the terms introduced by symmetry. $\mathscr{E}$ is defined to be the expectation operator with respect to the probability measure. $\|v\|$ is the Euclidean norm of vector $v$, $\|v\|=\left(v^{T} v\right)^{1 / 2}$, while $\|A\|$ is spectral norm of matrix $A$, $\|A\|=\left[\lambda_{\max }\left(A^{T} A\right)\right]^{1 / 2} \cdot \lambda_{\max (\min )}(A)$ is the eigenvalue of matrix $A$ with maximum (minimum) real part. Matrices, if their dimensions are not explicitly stated, are assumed to have compatible dimensions for algebraic operations.

\section{Problem Statement and Preliminaries}

Given a probability space $\{\Omega, \mathscr{F}, \mathbf{P}\}$ where $\Omega$ is the sample space, $\mathscr{F}$ is the algebra of events, and $\mathbf{P}$ is the probability measure defined on $\mathscr{F} .\left\{r_{t}, t \geq 0\right\}$ is a homogeneous, finitestate Markovian process with right continuous trajectories taking values in a finite set $S=\{1,2,3, \ldots, N\}$, with the mode transition probability matrix being

$$
P\left(r_{t+\Delta t}=j \mid r_{t}=i\right)= \begin{cases}\pi_{i j} \Delta t+o(\Delta t), & i \neq j, \\ 1+\pi_{i i} \Delta t+o(\Delta t), & i=j,\end{cases}
$$

where $\Delta t>0, \lim _{\Delta t \rightarrow 0}(o(\Delta t) / \Delta t)=0$, and $\pi_{i j} \geq 0(i, j \in$ $S, i \neq j)$ is the transition rate from mode $i$ to $j$, and for any state or mode $i \in S$, it satisfies

$$
\pi_{i i}=-\sum_{j=1, j \neq i}^{N} \pi_{i j}
$$

The following uncertain neutral Markovian jump systems with interval time-varying delays and nonlinear perturbations over the space $\{\Omega, \mathscr{F}, \mathbf{P}\}$ are considered:

$$
\begin{aligned}
\dot{x}(t) & -C\left(r_{t}\right) \dot{x}(t-\tau(t)) \\
= & {\left[A\left(r_{t}\right)+\Delta A\left(r_{t}\right)\right] x(t) } \\
& +\left[B\left(r_{t}\right)+\Delta B\left(r_{t}\right)\right] x(t-d(t)) \\
& +f_{1}(x(t), t)+f_{2}(x(t-d(t)), t) \\
& +f_{3}(\dot{x}(t-\tau(t)), t), \\
x(s) & =\varphi(s), \quad r_{s}=r_{0}, \quad s \in[-\rho, 0],
\end{aligned}
$$

where $x(t) \in \mathbb{R}^{n}$ is the system state and $\tau(t)$ is time-varying neutral delay which satisfies $0 \leq \tau(t) \leq \tau, \dot{\tau}(t) \leq \tau_{d}$. The time-varying retarded delay $d(t)$ is such that

$$
0<d_{1} \leq d(t) \leq d_{2}, \quad \dot{d}(t) \leq \mu,
$$

where $d_{1}<d_{2}, \mu \geq 0$, and $\rho=\max \left\{\tau, d_{2}\right\}$ are constant real values. The initial condition $\varphi(s)$ is a continuously differentiable vector-valued function. $f_{1}(x(t), t) \in \mathbb{R}^{n}$, 
$f_{2}(x(t-d(t)), t) \in \mathbb{R}^{n}$, and $f_{3}(\dot{x}(t-\tau(t)), t) \in \mathbb{R}^{n}$ are unknown nonlinear perturbations which are, with respect to the current state $x(t)$, the delayed state $x(t-d(t))$ and the neutral delay state $\dot{x}(t-\tau(t))$, respectively. For all $t$, they are assumed to be bounded in magnitude as

$$
\begin{gathered}
\left\|f_{1}(x(t), t)\right\| \leq \alpha\|x(t)\|, \\
\left\|f_{2}(x(t-d(t)), t)\right\| \leq \beta\|x(t-d(t))\|, \\
\left\|f_{3}(\dot{x}(t-\tau(t)), t)\right\| \leq \gamma\|\dot{x}(t-\tau(t))\|,
\end{gathered}
$$

where $\alpha \geq 0, \beta \geq 0$, and $\gamma \geq 0$ are given constants, for simplicity, $f_{1} \triangleq f_{1}(x(t), t), f_{2} \triangleq f_{2}(x(t-d(t)), t)$, and $f_{3} \triangleq f_{3}(\dot{x}(t-\tau(t)), t)$.

$A\left(r_{t}\right) \in \mathbb{R}^{n \times n}, B\left(r_{t}\right) \in \mathbb{R}^{n \times n}$, and $C\left(r_{t}\right) \in \mathbb{R}^{n \times n}$ are known mode-dependent constant matrices, and $\Delta A\left(r_{t}\right) \in \mathbb{R}^{n \times n}$, $\Delta B\left(r_{t}\right) \in \mathbb{R}^{n \times n}$ are uncertainties. For simplicity of notations, we denote $A\left(r_{t}\right), \Delta A\left(r_{t}\right), B\left(r_{t}\right), \Delta B\left(r_{t}\right), C\left(r_{t}\right)$ by $A_{i}, \Delta A_{i}, B_{i}$, $\Delta B_{i}, C_{i}$ for $r_{t}=i \in S$. The parametric matrix $\left\|C_{i}\right\|<1$ and the admissible parametric uncertainties satisfy the following condition:

$$
\left[\Delta A_{i}(t) \quad \Delta B_{i}(t)\right]=H_{i} F_{i}(t)\left[\begin{array}{ll}
E_{A i} & E_{B i}
\end{array}\right],
$$

where $H_{i}, E_{A i}$, and $E_{B i}$ are known mode-dependent constant matrices with appropriate dimensions and $F_{i}(t)$ is an unknown and time-varying matrix satisfying

$$
F_{i}^{T}(t) F_{i}(t) \leq I, \quad \forall t .
$$

Particularly, the following nominal systems can be obtained for $F_{i}(t)=0$ :

$$
\begin{aligned}
\dot{x}(t) & -C_{i} \dot{x}(t-\tau(t)) \\
& =A_{i} x(t)+B_{i} x(t-d(t))+f_{1}+f_{2}+f_{3} .
\end{aligned}
$$

Before proceeding with the main results, we present the following definitions, assumptions, and lemmas.

Assumption 1. System matrices $A_{i},(\forall i \in S)$ are Hurwitz, and all the eigenvalues have negative real parts for each mode. $H_{i}$, $(\forall i \in S)$ is full rank in row.

Assumption 2. The Markov process is irreducible, and the system mode $r_{t}$ is available at time $t$.

Definition 3 (see [28]). Define operator $\mathfrak{D}: C([-\rho, 0]$, $\left.\mathbb{R}^{n}\right) \rightarrow \mathbb{R}^{n}$ as $\mathfrak{D}\left(x_{t}\right)=x(t)-C x(t-\tau)$. $\mathfrak{D}$ is said to be stable if the homogeneous difference equation

$$
\begin{gathered}
\mathfrak{D}\left(x_{t}\right)=0, \quad t \geq 0, \\
x_{0}=\psi \in\left\{\phi \in C\left([-\rho, 0], \mathbb{R}^{n}\right): \mathfrak{D} \phi=0\right\}
\end{gathered}
$$

is uniformly asymptotically stable. In this paper, that is, $\left\|C_{i}\right\|+$ $\gamma<1$.

Definition 4 (see [29]). The system in (3) is exponentially stable with a decay rate $\varepsilon$ for all $r_{t}=i \in S$, if there exist scalars $\varepsilon>0$ and $\sigma \geq 1$ such that for all $x(t)$,

$$
\|x(t)\| \leq \sigma \exp \left\{-\varepsilon\left(t-t_{0}\right)\right\}\left\|x_{t_{0}}\right\|_{\rho},
$$

where $\varepsilon$ is the exponential decay rate, $\|*\|$ denotes the Euclidean norm, and

$$
\begin{aligned}
\left\|x_{t_{0}}\right\|_{\rho} & =\sup _{\theta \in[-\rho, 0]}\left\{\left\|x\left(t_{0}+\theta\right)\right\|,\left\|\dot{x}\left(t_{0}+\theta\right)\right\|\right\} \\
& =\sup _{s \in\left[-\rho, t_{0}\right]}\{\|\varphi(s)\|,\|\dot{\varphi}(s)\|\} .
\end{aligned}
$$

Definition 5 (see [30]). Define the stochastic LyapunovKrasovskii function of system (3) as $V\left(x(t), r_{t}=i, t>0\right)=$ $V\left(x_{t}, i, t\right)$, where its infinitesimal generator is defined as

$$
\begin{aligned}
& \mathfrak{L V}(x(t), i, t) \\
&= \lim _{\Delta t \rightarrow 0} \frac{1}{\Delta t}\left[\mathscr { E } \left\{V\left(x(t+\Delta t), r_{t+\Delta t}, t+\Delta t\right) \mid x(t)=x,\right.\right. \\
&\left.\left.\quad r_{t}=i\right\}-V(x(t), i, t)\right] \\
&=\frac{\partial}{\partial t} V(x(t), i, t)+\frac{\partial}{\partial x} V(x(t), i, t) \dot{x}(t) \\
& \quad+\sum_{j=1}^{N} \pi_{i j} V(x(t), j, t) .
\end{aligned}
$$

Lemma 6 (see [31]). For given real constant matrices $\Omega_{1}, \Omega_{2}$, and $\Omega_{3}$, with appropriate dimensions, where $\Omega_{1}=\Omega_{1}^{T}$ and $\Omega_{2}=\Omega_{2}^{T}>0 . \Omega_{1}+\Omega_{3}^{T} \Omega_{2}^{-1} \Omega_{3}<0$ if and only if

$$
\left[\begin{array}{cc}
\Omega_{1} & \Omega_{3}^{T} \\
* & -\Omega_{2}
\end{array}\right]<0 \quad \text { or } \quad\left[\begin{array}{cc}
-\Omega_{2} & \Omega_{3}^{T} \\
* & \Omega_{1}
\end{array}\right]<0 .
$$

Lemma 7. For any constant matrix $H=H^{T}>0$, continuous functions $0 \leq h_{1}(t) \leq h_{2}(t)$, constant scalars $0 \leq \tau_{1}<\tau_{2}$, and constant $\varepsilon>0$ such that the following integrations are well defined,

(a)

$$
\begin{aligned}
& \frac{\exp \left\{\varepsilon h_{2}(t)\right\}-\exp \left\{\varepsilon h_{1}(t)\right\}}{\varepsilon} \\
& \times \int_{t-h_{2}(t)}^{t-h_{1}(t)} \exp \{\varepsilon(s-t)\} x^{T}(s) H x(s) d s \\
& \quad \geq\left[\int_{t-h_{2}(t)}^{t-h_{1}(t)} x^{T}(s) d s\right] H\left[\int_{t-h_{2}(t)}^{t-h_{1}(t)} x(s) d s\right],
\end{aligned}
$$

(b)

$$
\begin{aligned}
& \frac{\tau_{2}^{2}-\tau_{1}^{2}}{2} \int_{-\tau_{2}}^{-\tau_{1}} \int_{t+\theta}^{t} \exp \{\varepsilon(s-t)\} x^{T}(s) H x(s) d s d \theta \\
& \geq \exp \left\{-\varepsilon \tau_{2}\right\}\left[\int_{-\tau_{2}}^{-\tau_{1}} \int_{t+\theta}^{t} x^{T}(s) d s d \theta\right] \\
& \times H\left[\int_{-\tau_{2}}^{-\tau_{1}} \int_{t+\theta}^{t} x(s) d s d \theta\right] .
\end{aligned}
$$


Proof. (a) Is directly obtained from [32]. In addition, from $-\tau_{2} \leq \theta \leq-\tau_{1}$ and $t+\theta \leq s \leq t$, it is held that $-\tau_{2} \leq \theta \leq$ $s-t \leq 0$. Then,

$$
\begin{aligned}
& \int_{-\tau_{2}}^{-\tau_{1}} \int_{t+\theta}^{t} \exp \{\varepsilon(s-t)\} x^{T}(s) H x(s) d s d \theta \\
& \quad \geq \exp \left\{-\varepsilon \tau_{2}\right\} \int_{-\tau_{2}}^{-\tau_{1}} \int_{t+\theta}^{t} x^{T}(s) H x(s) d s d \theta
\end{aligned}
$$

(b) Is thus true by [33].

Lemma 8 (see [34]). For functions $\lambda_{1}(t), \lambda_{2}(t) \in[0,1]$, $\lambda_{1}(t)+\lambda_{2}(t)=1$, and $\eta_{1}=0$ with $\lambda_{1}(t)=0$ and $\eta_{2}=0$ with $\lambda_{2}(t)=0$, matrices $P>0, Q>0$; then there exists matrix $T$ such that

$$
\left[\begin{array}{cc}
P & T \\
T^{T} & Q
\end{array}\right]>0
$$

and the following inequality holds:

$$
\frac{1}{\lambda_{1}(t)} \eta_{1} P \eta_{1}^{T}+\frac{1}{\lambda_{2}(t)} \eta_{2} Q \eta_{2}^{T} \geq\left[\begin{array}{ll}
\eta_{1} & \eta_{2}
\end{array}\right]\left[\begin{array}{cc}
P & T \\
T^{T} & Q
\end{array}\right]\left[\begin{array}{c}
\eta_{1}^{T} \\
\eta_{2}^{T}
\end{array}\right] .
$$

Lemma 9 (see [35]). For given matrices $Q=Q^{T}, M$, and $N$ with appropriate dimensions:

$$
Q+M F(t) N+N^{T} F^{T}(t) M^{T}<0,
$$

for all $F(t)$ satisfying $F^{T}(t) F(t) \leq I$, if and only if there exists a scalar $\delta>0$, such that

$$
Q+\delta^{-1} M M^{T}+\delta N N^{T}<0 .
$$

\section{Main Results}

This section will first state the exponential stability analysis for (9) with known and partly unknown transition rates, respectively. Then, the uncertain systems described by (3) are considered. With creative Lyapunov functional and novel matrix inequalities analysis, delay-range-dependent and ratedependent exponential stability conditions are presented.

3.1. Exponential Stability for the Nominal Systems with Known Transition Rates. In this subsection, we consider the full information on the transition rates and give the following conditions to guarantee the exponential stability of the nominal systems.

Theorem 10. For the given finite set $S$ of modes with transition rates matrix, scalars $\alpha, \beta, \gamma, \varepsilon, \tau, \tau_{d}, d_{1}, d_{2}, \mu$, and constant scalar $d_{m}$ satisfying $d_{1}<d_{m}<d_{2}$, the systems described by (9) are exponentially stable with decay rate $\varepsilon / 2$ and $\sigma=\sqrt{\bar{\lambda} / \lambda}$ if the operator $\mathfrak{D}$ is stable, and there exist symmetric positive matrices $P_{i}>0,(i \in S), Q_{j}>0,(j=1,2,3,4,5), R_{k}>0,(k=$ $1,2,3,4,5), W_{l}>0,(l=1,2,3,4), Y_{m}>0,(m=1,2,3,4)$,
$Z_{n}>0,(n=1,2,3,4)$ and matrices $U_{1}, U_{2}, X_{1}, X_{2}, X_{3}, X_{4}$ for any scalars $\varepsilon_{1}, \varepsilon_{2}, \varepsilon_{3}$ and any matrices $T_{k},(k=1,2, \ldots, 23)$ with appropriate dimensions, such that the following linear matrix inequalities (22), (23), and (24) hold:

$$
\left[\begin{array}{ll}
W_{1} & U_{1} \\
U_{1}^{T} & W_{1}
\end{array}\right]>0, \quad\left[\begin{array}{cc}
Y_{1} & U_{2} \\
U_{2}^{T} & Y_{1}
\end{array}\right]>0,
$$

(i) $\left[\begin{array}{ll}W_{3} & X_{1} \\ X_{1}^{T} & W_{3}\end{array}\right]>0, \quad\left[\begin{array}{cc}Y_{3} & X_{2} \\ X_{2}^{T} & Y_{3}\end{array}\right]>0$,

(ii) $\Xi_{i 0}^{\varepsilon}+\Xi_{i 1}^{\varepsilon}<0$,

(iii) $\left[\begin{array}{ll}W_{4} & X_{3} \\ X_{3}^{T} & W_{4}\end{array}\right]>0, \quad\left[\begin{array}{cc}Y_{4} & X_{4} \\ X_{4}^{T} & Y_{4}\end{array}\right]>0$,

(iv) $\Xi_{i 0}^{\varepsilon}+\Xi_{i 2}^{\varepsilon}<0$,

where

$$
\begin{aligned}
& \Xi_{i 0}^{\varepsilon}=\sum_{m=1}^{23} e_{m} G_{m} e_{m}^{T}+\mathscr{A}(\text { ( }) \\
& -\frac{\varepsilon d_{1}}{\exp \left\{\varepsilon d_{1}\right\}-1}\left(e_{1}-e_{4}\right) Y_{2}\left(e_{1}^{T}-e_{4}^{T}\right) \\
& -\exp \{-\varepsilon \tau\}\left(\tau e_{1}-e_{20}\right) Z_{1}\left(\tau e_{1}^{T}-e_{20}^{T}\right) \\
& -\exp \left\{-\varepsilon d_{1}\right\}\left(d_{1} e_{1}-e_{10}\right) Z_{2}\left(d_{1} e_{1}^{T}-e_{10}^{T}\right) \\
& -\exp \left\{-\varepsilon d_{m}\right\}\left(\varrho_{1} e_{1}-e_{13}\right) Z_{3}\left(\varrho_{1} e_{1}^{T}-e_{13}^{T}\right) \\
& -\exp \left\{-\varepsilon d_{2}\right\}\left(\varrho_{2} e_{1}-e_{14}\right) Z_{4}\left(\varrho_{2} e_{1}^{T}-e_{14}^{T}\right) \\
& -\frac{\varepsilon \tau}{\exp \{\varepsilon \tau\}-1}\left[\begin{array}{ll}
e_{19} & e_{20}-e_{19}
\end{array}\right]\left[\begin{array}{cc}
W_{1} & U_{1} \\
U_{1}^{T} & W_{1}
\end{array}\right]\left[\begin{array}{c}
e_{19}^{T} \\
e_{20}^{T}-e_{19}^{T}
\end{array}\right] \\
& -\frac{\varepsilon \tau}{\exp \{\varepsilon \tau\}-1}\left[\begin{array}{ll}
e_{1}-e_{17} & e_{17}-e_{15}
\end{array}\right] \\
& \times\left[\begin{array}{cc}
Y_{1} & U_{2} \\
U_{2}^{T} & Y_{1}
\end{array}\right]\left[\begin{array}{c}
e_{1}^{T}-e_{17}^{T} \\
e_{17}^{T}-e_{15}^{T}
\end{array}\right]
\end{aligned}
$$

where $\mathscr{A}$ is a linear operator on $\mathbb{R}^{n \times n}$ by

$$
\begin{gathered}
\mathscr{A}(F)=F+F^{T}, \quad \forall F \in \mathbb{R}^{n \times n}, \\
\mathbb{\Psi}=-e_{1} T_{1} e_{2}^{T}+e_{1}\left(P_{i} B_{i}+T_{1} B_{i}\right) e_{3}^{T}+e_{1}\left(P_{i} C_{i}\right) e_{18}^{T} \\
+\sum_{m=2}^{23} e_{1}\left(A_{i}^{T} T_{m}^{T}\right) e_{m}^{T}+\sum_{m=21}^{23} e_{1}\left(P_{i}\right) e_{m}^{T} \\
+e_{2}\left(T_{2} B_{i}\right) e_{3}^{T}-\sum_{m=3}^{23} e_{2}\left(T_{m}^{T}\right) e_{m}^{T}+\sum_{m=4}^{23} e_{3}\left(B_{i}^{T} T_{m}^{T}\right) e_{m}^{T}
\end{gathered}
$$




$$
\begin{aligned}
& +\sum_{m=1}^{17} e_{m}\left(T_{m} C_{i}\right) e_{18}^{T}+\sum_{m=19}^{23} e_{18}\left(C_{i}^{T} T_{m}^{T}\right) e_{m}^{T} \\
& +\sum_{m=1}^{20} \sum_{n=21}^{23} e_{m} T_{m} e_{n}^{T}+e_{21} T_{21}\left(e_{22}^{T}+e_{23}^{T}\right) \\
& +e_{22} T_{22} e_{23}^{T}+\sum_{m=22}^{23} e_{21} T_{m}^{T} e_{m}^{T}+e_{22} T_{23}^{T} e_{23}^{T}, \\
& G_{1}=A_{i}^{T} P_{i}+P_{i} A_{i}+\sum_{j=1}^{N} \pi_{i j} P_{j}+\varepsilon P_{i}+Q_{1} \\
& +Q_{3}+\tau^{2} W_{1}+d_{1}^{2} W_{2}+\varrho_{1}^{2} W_{3}+\varrho_{2}^{2} W_{4} \\
& +\varepsilon_{1} \alpha^{2} I+T_{1} A_{i}+A_{i}^{T} T_{1}^{T}, \\
& G_{2}=R_{1}+R_{2}+R_{5}+\tau^{2} Y_{1}+d_{1}^{2} Y_{2}+\varrho_{1}^{2} Y_{3}+\varrho_{2}^{2} Y_{4}+\frac{\tau^{4}}{4} Z_{1} \\
& +\frac{d_{1}^{4}}{4} Z_{2}+\varrho_{3}^{2} Z_{3}+\varrho_{4}^{2} Z_{4}-T_{2}-T_{2}^{T} \\
& G_{3}=\chi(\varepsilon, \mu) Q_{2}+T_{3} B_{i}+B_{i}^{T} T_{3}^{T}+\varepsilon_{2} \beta^{2} I, \\
& G_{4}=\exp \left\{-\varepsilon d_{1}\right\}\left(Q_{2}+Q_{4}-Q_{3}\right) \text {, } \\
& G_{5}=\exp \left\{-\varepsilon d_{m}\right\}\left(Q_{5}-Q_{4}\right), \\
& G_{6}=-\exp \left\{-\varepsilon d_{2}\right\} Q_{5}, \\
& G_{7}=\exp \left\{-\varepsilon d_{1}\right\}\left(R_{3}-R_{2}\right) \text {, } \\
& G_{8}=\exp \left\{-\varepsilon d_{m}\right\}\left(R_{4}-R_{3}\right), \\
& G_{9}=-\exp \left\{-\varepsilon d_{2}\right\} R_{4} \text {, } \\
& G_{10}=-\frac{\varepsilon d_{1}}{\exp \left\{\varepsilon d_{1}\right\}-1} W_{2}, \\
& G_{m}=0, \quad(m=11,12,13,14,17,19,20), \\
& G_{15}=-\exp \{-\varepsilon \tau\} Q_{1}, \\
& G_{16}=-\exp \{-\varepsilon \tau\} R_{1} \text {, } \\
& G_{18}=-\left(1-\tau_{d}\right) \exp \{-\varepsilon \tau \tau\} R_{5}+T_{18} C_{i}+C_{i}^{T} T_{18}^{T}+\varepsilon_{3} \gamma^{2} I \text {, } \\
& \begin{array}{l}
G_{21}=-\varepsilon_{1} I+T_{21}+T_{21}^{T}, \\
G_{22}=-\varepsilon_{2} I+T_{22}+T_{22}^{T}, \\
G_{23}=-\varepsilon_{3} I+T_{23}+T_{23}^{T},
\end{array} \\
& \Xi_{i 1}^{\varepsilon}=-\frac{\varepsilon \varrho_{2}}{\exp \left\{\varepsilon d_{2}\right\}-\exp \left\{\varepsilon d_{m}\right\}} e_{14} W_{4} e_{14}^{T} \\
& -\frac{\varepsilon \varrho_{2}}{\exp \left\{\varepsilon d_{2}\right\}-\exp \left\{\varepsilon d_{m}\right\}}\left(e_{5}-e_{6}\right) Y_{4}\left(e_{5}^{T}-e_{6}^{T}\right) \\
& -\frac{\varepsilon \varrho_{1}}{\exp \left\{\varepsilon d_{m}\right\}-\exp \left\{\varepsilon d_{1}\right\}}
\end{aligned}
$$$$
\begin{aligned}
& \times\left[\begin{array}{ll}
e_{11} & e_{13}-e_{11}
\end{array}\right]\left[\begin{array}{cc}
W_{3} & X_{1} \\
X_{1}^{T} & W_{3}
\end{array}\right]\left[\begin{array}{c}
e_{11}^{T} \\
e_{13}^{T}-e_{11}^{T}
\end{array}\right] \\
& -\frac{\varepsilon \varrho_{1}}{\exp \left\{\varepsilon d_{m}\right\}-\exp \left\{\varepsilon d_{1}\right\}} \\
& \times\left[e_{4}-e_{3} e_{3}-e_{5}\right]\left[\begin{array}{cc}
Y_{3} & X_{2} \\
X_{2}^{T} & Y_{3}
\end{array}\right]\left[\begin{array}{c}
e_{4}^{T}-e_{3}^{T} \\
e_{3}^{T}-e_{5}^{T}
\end{array}\right],
\end{aligned}
$$$$
\Xi_{i 2}^{\varepsilon}=-\frac{\varepsilon \varrho_{1}}{\exp \left\{\varepsilon d_{m}\right\}-\exp \left\{\varepsilon d_{1}\right\}} e_{13} W_{3} e_{13}^{T}
$$$$
-\frac{\varepsilon \varrho_{1}}{\exp \left\{\varepsilon d_{m}\right\}-\exp \left\{\varepsilon d_{1}\right\}}\left(e_{4}-e_{5}\right) Y_{3}\left(e_{4}^{T}-e_{5}^{T}\right)
$$$$
-\frac{\varepsilon \varrho_{2}}{\exp \left\{\varepsilon d_{2}\right\}-\exp \left\{\varepsilon d_{m}\right\}}
$$$$
\times\left[\begin{array}{ll}
e_{12} & e_{14}-e_{12}
\end{array}\right]\left[\begin{array}{ll}
W_{4} & X_{3} \\
X_{3}^{T} & W_{4}
\end{array}\right]\left[\begin{array}{c}
e_{12}^{T} \\
e_{14}^{T}-e_{12}^{T}
\end{array}\right]
$$$$
-\frac{\varepsilon \varrho_{2}}{\exp \left\{\varepsilon d_{2}\right\}-\exp \left\{\varepsilon d_{m}\right\}}
$$$$
\times\left[\begin{array}{ll}
e_{3}-e_{6} & e_{5}-e_{3}
\end{array}\right]\left[\begin{array}{cc}
Y_{4} & X_{4} \\
X_{4}^{T} & Y_{4}
\end{array}\right]\left[\begin{array}{c}
e_{3}^{T}-e_{6}^{T} \\
e_{5}^{T}-e_{3}^{T}
\end{array}\right],
$$

where $e_{i}\{i=1,2, \ldots, 23\}$ are block entry matrices; that is,

$$
e_{2}^{T}
$$

$=\left[\begin{array}{lllllllllllllllllllllll}0 & I & 0 & 0 & 0 & 0 & 0 & 0 & 0 & 0 & 0 & 0 & 0 & 0 & 0 & 0 & 0 & 0 & 0 & 0 & 0 & 0 & 0\end{array}\right]$,

$$
\chi(\varepsilon, \mu)=(\mu-1) \exp \left\{-\varepsilon d_{1}\right\} \text { with } \mu \geq 1,
$$$$
(\mu-1) \exp \left\{-\varepsilon d_{2}\right\} \text { with } \mu<1 \text {, }
$$

$$
\begin{aligned}
\bar{\lambda}= & \max _{i \in S}\left\{\lambda_{\max }\left(P_{i}\right)\right\} \\
& +\frac{1-\exp \{-\varepsilon \rho\}}{\varepsilon}\left\{\sum_{j=1}^{5} \lambda_{\max }\left(Q_{j}\right)+\sum_{k=1}^{5} \lambda_{\max }\left(R_{k}\right)\right\} \\
& +\frac{\exp \{-\varepsilon \rho\}+\varepsilon \rho-1}{\varepsilon^{2}} \\
& \times\left\{\sum_{l=1}^{4} \lambda_{\max }\left(W_{l}\right)+\sum_{m=1}^{4} \lambda_{\max }\left(Y_{m}\right)\right\} \\
& +\frac{2-2 \exp \{-\varepsilon \rho\}+\rho^{2} \varepsilon^{2}-2 \varepsilon \rho}{2 \varepsilon^{3}}\left\{\sum_{n=1}^{4} \lambda_{\max }\left(Z_{n}\right)\right\}, \\
& \lambda \underline{x}_{i \in S}\left\{\lambda_{\min }\left(P_{i}\right)\right\}, \\
& \varrho_{3}=\frac{1}{2}\left(d_{m}^{2}-d_{1}, \quad \varrho_{2}^{2}\right), \quad \varrho_{4}=\frac{1}{2}\left(d_{2}^{2}-d_{m}^{2}\right) .
\end{aligned}
$$


Proof. Construct the following Lyapunov functional:

$$
V(x(t), i, t)=\sum_{k=1}^{6} V_{k}\left(x_{t}, i\right)
$$

where

$$
\begin{aligned}
& V_{1}\left(x_{t}, i\right)=x^{T}(t) P_{i} x(t), \\
& V_{2}\left(x_{t}, i\right)=\int_{t-\tau}^{t} \exp \{\varepsilon(s-t)\} x^{T}(s) Q_{1} x(s) d s \\
& +\int_{t-d(t)}^{t-d_{1}} \exp \{\varepsilon(s-t)\} x^{T}(s) Q_{2} x(s) d s \\
& +\int_{t-d_{1}}^{t} \exp \{\varepsilon(s-t)\} x^{T}(s) Q_{3} x(s) d s \\
& +\int_{t-d_{m}}^{t-d_{1}} \exp \{\varepsilon(s-t)\} x^{T}(s) Q_{4} x(s) d s \\
& +\int_{t-d_{2}}^{t-d_{m}} \exp \{\varepsilon(s-t)\} x^{T}(s) Q_{5} x(s) d s, \\
& V_{3}\left(x_{t}, i\right)=\int_{t-\tau}^{t} \exp \{\varepsilon(s-t)\} \dot{x}^{T}(s) R_{1} \dot{x}(s) d s \\
& +\int_{t-d_{1}}^{t} \exp \{\varepsilon(s-t)\} \dot{x}^{T}(s) R_{2} \dot{x}(s) d s \\
& +\int_{t-d_{m}}^{t-d_{1}} \exp \{\varepsilon(s-t)\} \dot{x}^{T}(s) R_{3} \dot{x}(s) d s \\
& +\int_{t-d_{2}}^{t-d_{m}} \exp \{\varepsilon(s-t)\} \dot{x}^{T}(s) R_{4} \dot{x}(s) d s \\
& +\int_{t-\tau(t)}^{t} \exp \{\varepsilon(s-t)\} \dot{x}^{T}(s) R_{5} \dot{x}(s) d s,
\end{aligned}
$$

$$
V_{4}\left(x_{t}, i\right)=\int_{-\tau}^{0} \int_{t+\theta}^{t} \tau \exp \{\varepsilon(s-t)\} x^{T}(s) W_{1} x(s) d s d \theta
$$$$
+\int_{-d_{1}}^{0} \int_{t+\theta}^{t} d_{1} \exp \{\varepsilon(s-t)\} x^{T}(s) W_{2} x(s) d s d \theta
$$$$
+\int_{-d_{m}}^{-d_{1}} \int_{t+\theta}^{t} \varrho_{1} \exp \{\varepsilon(s-t)\} x^{T}(s) W_{3} x(s) d s d \theta
$$$$
+\int_{-d_{2}}^{-d_{m}} \int_{t+\theta}^{t} \varrho_{2} \exp \{\varepsilon(s-t)\} x^{T}(s) W_{4} x(s) d s d \theta,
$$

$$
\begin{aligned}
V_{5}\left(x_{t}, i\right)= & \int_{-\tau}^{0} \int_{t+\theta}^{t} \tau \exp \{\varepsilon(s-t)\} \dot{x}^{T}(s) Y_{1} \dot{x}(s) d s d \theta \\
& +\int_{-d_{1}}^{0} \int_{t+\theta}^{t} d_{1} \exp \{\varepsilon(s-t)\} \dot{x}^{T}(s) Y_{2} \dot{x}(s) d s d \theta
\end{aligned}
$$

$$
\begin{aligned}
& +\int_{-d_{m}}^{-d_{1}} \int_{t+\theta}^{t} \varrho_{1} \exp \{\varepsilon(s-t)\} \dot{x}^{T}(s) Y_{3} \dot{x}(s) d s d \theta \\
& +\int_{-d_{2}}^{-d_{m}} \int_{t+\theta}^{t} \varrho_{2} \exp \{\varepsilon(s-t)\} \dot{x}^{T}(s) Y_{4} \dot{x}(s) d s d \theta,
\end{aligned}
$$

$$
\begin{aligned}
V_{6}\left(x_{t}, i\right) \\
=\int_{-\tau}^{0} \int_{\theta}^{0} \int_{t+\lambda}^{t} \frac{\tau^{2}}{2} \exp \{\varepsilon(s-t)\} \dot{x}^{T}(s) Z_{1} \dot{x}(s) d s d \lambda d \theta \\
\quad+\int_{-d_{1}}^{0} \int_{\theta}^{0} \int_{t+\lambda}^{t} \frac{d_{1}^{2}}{2} \exp \{\varepsilon(s-t)\} \dot{x}^{T}(s) Z_{2} \dot{x}(s) d s d \lambda d \theta \\
\quad+\int_{-d_{m}}^{-d_{1}} \int_{\theta}^{0} \int_{t+\lambda}^{t} \varrho_{3} \exp \{\varepsilon(s-t)\} \dot{x}^{T}(s) Z_{3} \dot{x}(s) d s d \lambda d \theta \\
\quad+\int_{-d_{2}}^{-d_{m}} \int_{\theta}^{0} \int_{t+\lambda}^{t} \varrho_{4} \exp \{\varepsilon(s-t)\} \dot{x}^{T}(s) Z_{4} \dot{x}(s) d s d \lambda d \theta .
\end{aligned}
$$

Taking $\mathfrak{Q}$ as its infinitesimal generator along the trajectory of system (9), we obtain the following from Definition 5 and (28)-(34):

$$
\begin{aligned}
\mathfrak{Q} V(x(t), i, t)= & \mathfrak{Q} V_{1}\left(x_{t}, i\right)+\mathfrak{Q} V_{2}\left(x_{t}, i\right)+\mathfrak{Q} V_{3}\left(x_{t}, i\right) \\
& +\mathfrak{Q} V_{4}\left(x_{t}, i\right)+\mathfrak{Q} V_{5}\left(x_{t}, i\right)+\mathfrak{Q} V_{6}\left(x_{t}, i\right),
\end{aligned}
$$

$$
\begin{aligned}
\mathfrak{Q} V_{1}\left(x_{t}, i\right)=2[ & x^{T}(t) A_{i}^{T}+x^{T}(t-d(t)) B_{i}^{T} \\
& \left.+\dot{x}^{T}(t-\tau(t)) C_{i}^{T}+f_{1}^{T}+f_{2}^{T}+f_{3}^{T}\right] \\
\times & P_{i} x(t)+\sum_{j=1}^{N} \pi_{i j} x^{T}(t) P_{j} x(t) \\
& +\varepsilon x^{T}(t) P_{i} x(t)-\varepsilon V_{1}\left(x_{t}, i\right),
\end{aligned}
$$

$$
\begin{aligned}
\mathcal{Q} V_{2}\left(x_{t}, i\right)= & x^{T}(t)\left[Q_{1}+Q_{3}\right] x(t)+\exp \left\{-\varepsilon d_{1}\right\} x^{T} \\
& \times\left(t-d_{1}\right)\left[Q_{2}+Q_{4}-Q_{3}\right] x\left(t-d_{1}\right) \\
& -\varepsilon V_{2}\left(x_{t}, i\right) \\
& -(1-\dot{d}(t)) \exp \{-\varepsilon d(t)\} x^{T}(t-d(t)) \\
& \times Q_{2} x(t-d(t))-\exp \{-\varepsilon \tau\} x^{T}(t-\tau) \\
& \times Q_{1} x(t-\tau)+\exp \left\{-\varepsilon d_{m}\right\} \\
& \times x^{T}\left(t-d_{m}\right)\left[Q_{5}-Q_{4}\right] \\
& \times x\left(t-d_{m}\right) \\
& -\exp \left\{-\varepsilon d_{2}\right\} x^{T}\left(t-d_{2}\right) Q_{5} x\left(t-d_{2}\right),
\end{aligned}
$$




$$
\begin{aligned}
\mathfrak{E} V_{3}\left(x_{t}, i\right)= & \dot{x}^{T}(t)\left[R_{1}+R_{2}+R_{5}\right] \dot{x}(t) \\
& +\exp \left\{-\varepsilon d_{1}\right\} \dot{x}^{T}\left(t-d_{1}\right)\left[R_{3}-R_{2}\right] \\
& \times \dot{x}\left(t-d_{1}\right)-\varepsilon V_{3}\left(x_{t}, i\right) \\
& +\exp \left\{-\varepsilon d_{m}\right\} \dot{x}^{T}\left(t-d_{m}\right)\left[R_{4}-R_{3}\right] \\
& \times \dot{x}\left(t-d_{m}\right)-\exp \{-\varepsilon \tau\} \\
& \times \dot{x}^{T}(t-\tau) R_{1} \dot{x}(t-\tau) \\
& -(1-\dot{\tau}(t)) \exp \{-\varepsilon \tau(t)\} \\
& \times \dot{x}^{T}(t-\tau(t)) R_{5} \dot{x}(t-\tau(t)) \\
& -\exp \left\{-\varepsilon d_{2}\right\} \dot{x}^{T}\left(t-d_{2}\right) R_{4} \dot{x}\left(t-d_{2}\right),
\end{aligned}
$$

$$
\begin{aligned}
\mathcal{S} V_{4}\left(x_{t}, i\right)= & x^{T}(t)\left[\tau^{2} W_{1}+d_{1}^{2} W_{2}+\varrho_{1}^{2} W_{3}+\varrho_{2}^{2} W_{4}\right] \\
& \times x(t)-\varepsilon V_{4}\left(x_{t}, i\right) \\
& -\int_{t-\tau}^{t} \tau \exp \{\varepsilon(s-t)\} x^{T}(s) W_{1} x(s) d s \\
& -\int_{t-d_{1}}^{t} d_{1} \exp \{\varepsilon(s-t)\} x^{T}(s) W_{2} x(s) d s \\
& -\int_{t-d_{m}}^{t-d_{1}} \varrho_{1} \exp \{\varepsilon(s-t)\} x^{T}(s) W_{3} x(s) d s \\
& -\int_{t-d_{2}}^{t-d_{m}} \varrho_{2} \exp \{\varepsilon(s-t)\} x^{T}(s) W_{4} x(s) d s,
\end{aligned}
$$

$$
\begin{aligned}
\mathfrak{S} V_{5}\left(x_{t}, i\right)= & \dot{x}^{T}(t)\left[\tau^{2} Y_{1}+d_{1}^{2} Y_{2}+\varrho_{1}^{2} Y_{3}+\varrho_{2}^{2} Y_{4}\right] \dot{x}(t) \\
& -\varepsilon V_{5}\left(x_{t}, i\right) \\
& -\int_{t-\tau}^{t} \tau \exp \{\varepsilon(s-t)\} \dot{x}^{T}(s) Y_{1} \dot{x}(s) d s \\
& -\int_{t-d_{1}}^{t} d_{1} \exp \{\varepsilon(s-t)\} \dot{x}^{T}(s) Y_{2} \dot{x}(s) d s \\
& -\int_{t-d_{m}}^{t-d_{1}} \varrho_{1} \exp \{\varepsilon(s-t)\} \dot{x}^{T}(s) Y_{3} \dot{x}(s) d s \\
& -\int_{t-d_{2}}^{t-d_{m}} \varrho_{2} \exp \{\varepsilon(s-t)\} \dot{x}^{T}(s) Y_{4} \dot{x}(s) d s
\end{aligned}
$$

$$
\begin{aligned}
\mathfrak{Q} V_{6}\left(x_{t}, i\right)= & \dot{x}^{T}(t) \\
& \times\left[\frac{\tau^{4}}{4} Z_{1}+\frac{d_{1}^{4}}{4} Z_{2}+\varrho_{3}^{2} Z_{3}+\varrho_{4}^{2} Z_{4}\right] \dot{x}(t) \\
& -\varepsilon V_{6}\left(x_{t}, i\right)
\end{aligned}
$$

$$
\begin{gathered}
-\int_{-\tau}^{0} \int_{t+\theta}^{t} \frac{\tau^{2}}{2} \exp \{\varepsilon(s-t)\} \dot{x}^{T}(s) \\
\times Z_{1} \dot{x}(s) d s d \theta \\
-\int_{-d_{1}}^{0} \int_{t+\theta}^{t} \frac{d_{1}^{2}}{2} \exp \{\varepsilon(s-t)\} \dot{x}^{T}(s) \\
\times Z_{2} \dot{x}(s) d s d \theta \\
-\int_{-d_{m}}^{-d_{1}} \int_{t+\theta}^{t} \varrho_{3} \exp \{\varepsilon(s-t)\} \dot{x}^{T}(s) \\
\times Z_{3} \dot{x}(s) d s d \theta \\
-\int_{-d_{2}}^{-d_{m}} \int_{t+\theta}^{t} \varrho_{4} \exp \{\varepsilon(s-t)\} \dot{x}^{T}(s) \\
\times Z_{4} \dot{x}(s) d s d \theta .
\end{gathered}
$$

In view of (6), the following inequalities hold for any scalars $\varepsilon_{1}>0, \varepsilon_{2}>0$, and $\varepsilon_{3}>0$ :

$$
\begin{aligned}
& \varepsilon_{1}\left[\alpha^{2} x^{T}(t) x(t)-f_{1}^{T}(x(t), t) f_{1}(x(t), t)\right] \geq 0, \\
& \varepsilon_{2}\left[\beta^{2} x^{T}(t-d(t)) x(t-d(t))\right. \\
& \left.\quad-f_{2}^{T}(x(t-d(t)), t) f_{2}(x(t-d(t)), t)\right] \geq 0, \\
& \varepsilon_{3}\left[\gamma^{2} \dot{x}^{T}(t-\tau(t)) \dot{x}(t-\tau(t))\right. \\
& \left.\quad-f_{3}^{T}(\dot{x}(t-\tau(t)), t) f_{3}(\dot{x}(t-\tau(t)), t)\right] \geq 0 .
\end{aligned}
$$

From (35) and (42), we have

$$
\begin{aligned}
\mathfrak{Q} V(x(t), i, t) \leq & \mathfrak{Q} V_{1}\left(x_{t}, i\right)+\mathfrak{Q} V_{2}\left(x_{t}, i\right)+\mathfrak{Q} V_{3}\left(x_{t}, i\right) \\
& +\mathfrak{Q} V_{4}\left(x_{t}, i\right)+\mathfrak{Q} V_{5}\left(x_{t}, i\right)+\mathfrak{Q} V_{6}\left(x_{t}, i\right) \\
& +\varepsilon_{1}\left[\alpha^{2} x^{T}(t) x(t)-f_{1}^{T} f_{1}\right] \\
& +\varepsilon_{2}\left[\beta^{2} x^{T}(t-d(t)) x(t-d(t))-f_{2}^{T} f_{2}\right] \\
& +\varepsilon_{3}\left[\gamma^{2} \dot{x}^{T}(t-\tau(t)) \dot{x}(t-\tau(t))-f_{3}^{T} f_{3}\right] .
\end{aligned}
$$

Define

$$
\begin{gathered}
\xi(t)=\operatorname{col}\left\{x(t) \dot{x}(t) x(t-d(t)) x\left(t-d_{1}\right) x\left(t-d_{m}\right) x\left(t-d_{2}\right) \dot{x}\left(t-d_{1}\right) \dot{x}\left(t-d_{m}\right) \dot{x}\left(t-d_{2}\right)\right. \\
\int_{t-d_{1}}^{t} x(s) d s \int_{t-d(t)}^{t-d_{1}} x(s) d s \int_{t-d_{2}}^{t-d(t)} x(s) d s \int_{t-d_{m}}^{t-d_{1}} x(s) d s \int_{t-d_{2}}^{t-d_{m}} x(s) d s \\
\left.x(t-\tau) \dot{x}(t-\tau) x(t-\tau(t)) \dot{x}(t-\tau(t)) \int_{t-\tau(t)}^{t} x(s) d s \int_{t-\tau}^{t} x(s) d s f_{1} f_{2} f_{3}\right\} .
\end{gathered}
$$


Since it is easy to see $-(1-\dot{\tau}(t)) \exp \{-\varepsilon \tau(t)\} \leq-\left(1-\tau_{d}\right)$ $\exp \{-\varepsilon \tau\}$; then from (5) we also obtain that

$$
-(1-\dot{d}(t)) \exp \{-\varepsilon d(t)\} \leq \chi(\varepsilon, \mu),
$$

where $\chi(\varepsilon, \mu)$ is defined in Theorem 10. Notice (a) of Lemma 7; then

$$
\begin{aligned}
& -\int_{t-d_{1}}^{t} d_{1} \exp \{\varepsilon(s-t)\} x^{T}(s) W_{2} x(s) d s \\
& \leq-\frac{\varepsilon d_{1}}{\exp \left\{\varepsilon d_{1}\right\}-1} \xi^{T}(t) e_{10} W_{2} e_{10}^{T} \xi(t), \\
& -\int_{t-d_{1}}^{t} d_{1} \exp \{\varepsilon(s-t)\} \dot{x}^{T}(s) Y_{2} \dot{x}(s) d s \\
& \leq-\frac{\varepsilon d_{1}}{\exp \left\{\varepsilon d_{1}\right\}-1} \xi^{T}(t)\left(e_{1}-e_{4}\right) Y_{2}\left(e_{1}^{T}-e_{4}^{T}\right) \xi(t) .
\end{aligned}
$$

Notice (b) of Lemma 7; then

$$
\begin{aligned}
& -\int_{-\tau}^{0} \int_{t+\theta}^{t} \frac{\tau^{2}}{2} \exp \{\varepsilon(s-t)\} \dot{x}^{T}(s) Z_{1} \dot{x}(s) d s d \theta \\
& \leq-\exp \{-\varepsilon \tau\} \xi^{T}(t)\left(\tau e_{1}-e_{20}\right) Z_{1}\left(\tau e_{1}^{T}-e_{20}^{T}\right) \xi(t), \\
& -\int_{-d_{1}}^{0} \int_{t+\theta}^{t} \frac{d_{1}^{2}}{2} \exp \{\varepsilon(s-t)\} \dot{x}^{T}(s) Z_{2} \dot{x}(s) d s d \theta \\
& \leq-\exp \left\{-\varepsilon d_{1}\right\} \xi^{T}(t)\left(d_{1} e_{1}-e_{10}\right) Z_{2}\left(d_{1} e_{1}^{T}-e_{10}^{T}\right) \xi(t), \\
& -\int_{-d_{m}}^{-d_{1}} \int_{t+\theta}^{t} \varrho_{3} \exp \{\varepsilon(s-t)\} \dot{x}^{T}(s) Z_{3} \dot{x}(s) d s d \theta \\
& \leq-\exp \left\{-\varepsilon d_{m}\right\} \xi^{T}(t)\left(\varrho_{1} e_{1}-e_{13}\right) Z_{3}\left(\varrho_{1} e_{1}^{T}-e_{13}^{T}\right) \xi(t), \\
& -\int_{-d_{2}}^{-d_{m}} \int_{t+\theta}^{t} \varrho_{4} \exp \{\varepsilon(s-t)\} \dot{x}^{T}(s) Z_{4} \dot{x}(s) d s d \theta \\
& \leq-\exp \left\{-\varepsilon d_{2}\right\} \xi^{T}(t)\left(\varrho_{2} e_{1}-e_{14}\right) Z_{4}\left(\varrho_{2} e_{1}^{T}-e_{14}^{T}\right) \xi(t) .
\end{aligned}
$$

For $d(t) \in\left[d_{1}, d_{m}\right]$, the following is held from (a) of Lemma 7:

$$
\begin{aligned}
& -\int_{t-d_{m}}^{t-d_{1}} \varrho_{1} \exp \{\varepsilon(s-t)\} \dot{x}^{T}(s) Y_{3} \dot{x}(s) d s \\
& \quad=-\left\{\int_{t-d(t)}^{t-d_{1}}+\int_{t-d_{m}}^{t-d(t)}\right\} \varrho_{1} \exp \{\varepsilon(s-t)\} \dot{x}^{T}(s) Y_{3} \dot{x}(s) d s
\end{aligned}
$$

$$
\begin{aligned}
\leq & -\frac{\varepsilon \varrho_{1}}{\exp \{\varepsilon d(t)\}-\exp \left\{\varepsilon d_{1}\right\}}\left[\int_{t-d(t)}^{t-d_{1}} \dot{x}^{T}(s) d s\right] \\
& \times Y_{3}\left[\int_{t-d(t)}^{t-d_{1}} \dot{x}(s) d s\right] \\
& -\frac{\varepsilon \varrho_{1}}{\exp \left\{\varepsilon d_{m}\right\}-\exp \{\varepsilon d(t)\}}\left[\int_{t-d_{m}}^{t-d(t)} \dot{x}^{T}(s) d s\right] \\
\times & Y_{3}\left[\int_{t-d_{m}}^{t-d(t)} \dot{x}(s) d s\right] \\
= & \frac{\varepsilon \varrho_{1}}{\exp \left\{\varepsilon d_{m}\right\}-\exp \left\{\varepsilon d_{1}\right\}} \xi^{T}(t) \\
\times & {\left[\frac{1}{\lambda_{1}(t)}\left(e_{4}-e_{3}\right) Y_{3}\left(e_{4}^{T}-e_{3}^{T}\right)\right.} \\
& \left.+\frac{1}{\lambda_{2}(t)}\left(e_{3}-e_{5}\right) Y_{3}\left(e_{3}^{T}-e_{5}^{T}\right)\right] \xi(t),
\end{aligned}
$$

where

$$
\begin{aligned}
& \lambda_{1}(t)=\frac{\exp \{\varepsilon d(t)\}-\exp \left\{\varepsilon d_{1}\right\}}{\exp \left\{\varepsilon d_{m}\right\}-\exp \left\{\varepsilon d_{1}\right\}}, \\
& \lambda_{2}(t)=\frac{\exp \left\{\varepsilon d_{m}\right\}-\exp \{\varepsilon d(t)\}}{\exp \left\{\varepsilon d_{m}\right\}-\exp \left\{\varepsilon d_{1}\right\}} .
\end{aligned}
$$

By Lemma 8 , there exists matrix $X_{2}$ with appropriate dimensions such that

$$
\begin{aligned}
-\int_{t-d_{m}}^{t-d_{1}} \varrho_{1} \exp \{\varepsilon(s-t)\} \dot{x}^{T}(s) Y_{3} \dot{x}(s) d s & \\
\leq & -\frac{\varepsilon \varrho_{1}}{\exp \left\{\varepsilon d_{m}\right\}-\exp \left\{\varepsilon d_{1}\right\}} \xi^{T}(t) \\
& \times\left[e_{4}-e_{3} e_{3}-e_{5}\right]\left[\begin{array}{cc}
Y_{3} & X_{2} \\
X_{2}^{T} & Y_{3}
\end{array}\right]\left[\begin{array}{c}
e_{4}^{T}-e_{3}^{T} \\
e_{3}^{T}-e_{5}^{T}
\end{array}\right] \xi(t), \\
& {\left[\begin{array}{cc}
Y_{3} & X_{2} \\
X_{2}^{T} & Y_{3}
\end{array}\right]>0 . }
\end{aligned}
$$

Similarly, considering $-\int_{t-d_{m}}^{t-d_{1}} \varrho_{1} \exp \{\varepsilon(s-t)\} x^{T}(s) W_{3}$ $x(s) d s$ and following the same procedure, there exists matrix $X_{1}$ with appropriate dimensions such that

$$
\begin{aligned}
& -\int_{t-d_{m}}^{t-d_{1}} \varrho_{1} \exp \{\varepsilon(s-t)\} x^{T}(s) W_{3} x(s) d s \\
\leq & -\frac{\varepsilon \varrho_{1}}{\exp \left\{\varepsilon d_{m}\right\}-\exp \left\{\varepsilon d_{1}\right\}} \xi^{T}(t) \\
& \times\left[\begin{array}{ll}
e_{11} & e_{13}-e_{11}
\end{array}\right]\left[\begin{array}{ll}
W_{3} & X_{1} \\
X_{1}^{T} & W_{3}
\end{array}\right]\left[\begin{array}{c}
e_{11}^{T} \\
e_{13}^{T}-e_{11}^{T}
\end{array}\right] \xi(t), \\
& {\left[\begin{array}{ll}
W_{3} & X_{1} \\
X_{1}^{T} & W_{3}
\end{array}\right]>0 . }
\end{aligned}
$$


For $\tau(t) \in[0, \tau]$, with the same matrix inequalities technique, we obtain the following:

$$
\begin{aligned}
& -\int_{t-\tau}^{t} \tau \exp \{\varepsilon(s-t)\} x^{T}(s) W_{1} x(s) d s \\
& \leq-\frac{\varepsilon \tau}{\exp \{\varepsilon \tau\}-1} \xi^{T}(t) \\
& \times\left[\begin{array}{ll}
e_{19} & e_{20}-e_{19}
\end{array}\right]\left[\begin{array}{cc}
W_{1} & U_{1} \\
U_{1}^{T} & W_{1}
\end{array}\right]\left[\begin{array}{c}
e_{19}^{T} \\
e_{20}^{T}-e_{19}^{T}
\end{array}\right] \xi(t), \\
& {\left[\begin{array}{ll}
W_{1} & U_{1} \\
U_{1}^{T} & W_{1}
\end{array}\right]>0} \\
& -\int_{t-\tau}^{t} \tau \exp \{\varepsilon(s-t)\} \dot{x}^{T}(s) Y_{1} \dot{x}(s) d s \\
& \leq-\frac{\varepsilon \tau}{\exp \{\varepsilon \tau\}-1} \xi^{T}(t) \\
& \times\left[e_{1}-e_{17} e_{17}-e_{15}\right]\left[\begin{array}{cc}
Y_{1} & U_{2} \\
U_{2}^{T} & Y_{1}
\end{array}\right]\left[\begin{array}{c}
e_{1}^{T}-e_{17}^{T} \\
e_{17}^{T}-e_{15}^{T}
\end{array}\right] \xi(t), \\
& {\left[\begin{array}{cc}
Y_{1} & U_{2} \\
U_{2}^{T} & Y_{1}
\end{array}\right]>0}
\end{aligned}
$$

Consider $-\int_{t-d_{2}}^{t-d_{m}} \varrho_{2} \exp \{\varepsilon(s-t)\} x^{T}(s) W_{4} x(s) d s$ and $-\int_{t-d_{2}}^{t-d_{m}} \varrho_{2} \exp \{\varepsilon(s-t)\} \dot{x}^{T}(s) Y_{4} \dot{x}(s) d s$, which are directly estimated by (a) of Lemma 7; that is,

$$
\begin{aligned}
& -\int_{t-d_{2}}^{t-d_{m}} \varrho_{2} \exp \{\varepsilon(s-t)\} x^{T}(s) W_{4} x(s) d s \\
& \leq-\frac{\varepsilon \varrho_{2}}{\exp \left\{\varepsilon d_{2}\right\}-\exp \left\{\varepsilon d_{m}\right\}} \xi^{T}(t) e_{14} W_{4} e_{14}^{T} \xi(t), \\
& -\int_{t-d_{2}}^{t-d_{m}} \varrho_{2} \exp \{\varepsilon(s-t)\} \dot{x}^{T}(s) Y_{4} \dot{x}(s) d s \\
& \leq-\frac{\varepsilon \varrho_{2}}{\exp \left\{\varepsilon d_{2}\right\}-\exp \left\{\varepsilon d_{m}\right\}} \xi^{T}(t) \\
& \quad \times\left(e_{5}-e_{6}\right) Y_{4}\left(e_{5}^{T}-e_{6}^{T}\right) \xi(t) .
\end{aligned}
$$

In addition, there exist matrices $T=\operatorname{col}\left\{T_{k},(k=1\right.$, $2, \ldots, 23)\}$ with appropriate dimensions, such that the following equality holds according to (9):

$$
\begin{aligned}
2 \xi^{T}(t) T[ & -\dot{x}(t)+A_{i} x(t)+B_{i} x(t-d(t)) \\
& \left.+C_{i} \dot{x}(t-\tau(t))+f_{1}+f_{2}+f_{3}\right]=0 .
\end{aligned}
$$

Substituting (36)-(42) and (45)-(54) into (43), we obtain $\mathfrak{Q} V(x(t), i, t)+\varepsilon V(x(t), i, t) \leq \xi^{T}(t)\left(\Xi_{i 0}^{\varepsilon}+\Xi_{i 1}^{\varepsilon}\right) \xi(t)$.

On the other hand, for $d(t) \in\left[d_{m}, d_{2}\right]$, the integral terms

$$
\begin{gathered}
-\int_{t-d_{2}}^{t-d_{m}} \varrho_{2} \exp \{\varepsilon(s-t)\} x^{T}(s) W_{4} x(s) d s, \\
-\int_{t-d_{2}}^{t-d_{m}} \varrho_{2} \exp \{\varepsilon(s-t)\} \dot{x}^{T}(s) Y_{4} \dot{x}(s) d s .
\end{gathered}
$$

The above equations: are disposed and estimated by Lemma 8.

$$
\begin{aligned}
& -\int_{t-d_{m}}^{t-d_{1}} \varrho_{1} \exp \{\varepsilon(s-t)\} x^{T}(s) W_{3} x(s) d s, \\
& -\int_{t-d_{m}}^{t-d_{1}} \varrho_{1} \exp \{\varepsilon(s-t)\} \dot{x}^{T}(s) Y_{3} \dot{x}(s) d s
\end{aligned}
$$

are directly estimated by (a) of Lemma 7. Therefore,

$$
\mathfrak{Q V}(x(t), i, t)+\varepsilon V(x(t), i, t) \leq \xi^{T}(t)\left(\Xi_{i 0}^{\varepsilon}+\Xi_{i 2}^{\varepsilon}\right) \xi(t) .
$$

With (55) and (58), the following inequality (59) is held for $d(t) \in\left[d_{1}, d_{2}\right]$ if (22), (23), and (24) are satisfied

$$
\mathfrak{Q} V(x(t), i, t)+\varepsilon V(x(t), i, t)<0 .
$$

From the Lyapunov functional (28) and (59), it is held that

$$
\begin{aligned}
& V(x(t), i, t) \geq \min _{i \in S}\left\{\lambda_{\min }\left(P_{i}\right)\right\}\|x(t)\|^{2}=\underline{\lambda}\|x(t)\|^{2}, \\
& V(x(t), i, t)<\exp \left\{-\varepsilon\left(t-t_{0}\right)\right\} V\left(x_{t_{0}}, r_{t_{0}}, t_{0}\right) .
\end{aligned}
$$

Moreover, we have

$$
V\left(x_{t_{0}}, r_{t_{0}}, t_{0}\right)=\sum_{k=1}^{6} V_{k}\left(x_{t_{0}}, r_{t_{0}}\right) \leq \bar{\lambda}\left\|x_{t_{0}}\right\|_{\rho}^{2} .
$$

Then, from (60) and (61), it is readily seen that

$$
\|x(t)\| \leq \sqrt{\frac{\bar{\lambda}}{\lambda}} \exp \left\{-\frac{\varepsilon}{2}\left(t-t_{0}\right)\right\}\left\|x_{t_{0}}\right\|_{\rho},
$$

where $\sigma=\sqrt{\bar{\lambda} / \underline{\lambda}} \geq 1$.

Therefore, by Definition 4 , the system (9) is exponentially stable with a decay rate $\varepsilon / 2$. This completes the proof. 
Remark 11. It is noted that the integral intervals in (61) are enlarged as follows:

$$
\begin{aligned}
& \sum_{k=1}^{6} V_{k}\left(x_{t_{0}}, r_{t_{0}}\right) \leq x^{T}\left(t_{0}\right) P_{i} x\left(t_{0}\right) \\
& +\int_{t_{0}-\rho}^{t_{0}} \exp \left\{\varepsilon\left(s-t_{0}\right)\right\} x^{T}(s)\left(\sum_{j=1}^{5} Q_{j}\right) x(s) d s \\
& +\int_{t_{0}-\rho}^{t_{0}} \exp \left\{\varepsilon\left(s-t_{0}\right)\right\} \dot{x}^{T}(s)\left(\sum_{k=1}^{5} R_{k}\right) \dot{x}(s) d s \\
& +\int_{t-\rho}^{0} \int_{t_{0}+\theta}^{t_{0}} \rho \exp \left\{\varepsilon\left(s-t_{0}\right)\right\} \\
& +\int_{-\rho}^{0} \int_{t_{0}+\theta}^{t_{0}} \rho \exp \left\{\varepsilon\left(s-t_{0}\right)\right\} \\
& +\int_{-\rho}^{0} \int_{\theta}^{0} \int_{t_{0}+\lambda}^{t_{0}} \frac{\rho^{2}}{2} \exp \left\{\varepsilon(s)\left(\sum_{l=1}^{4} W_{l}\right) x(s) d s d \theta\right. \\
& \times \dot{x}^{T}(s)\left(\sum_{m=1}^{4} Y_{m}\right) \dot{x}(s) d s d \theta
\end{aligned}
$$

Equation (61) can be obtained by letting $\bar{\lambda}$ be defined as previously.

Remark 12. In Theorem 10, the factors

$$
\begin{aligned}
& \frac{\exp \left\{\varepsilon d_{2}\right\}-\exp \{\varepsilon d(t)\}}{\varepsilon}, \\
& \frac{\exp \{\varepsilon d(t)\}-\exp \left\{\varepsilon d_{1}\right\}}{\varepsilon}
\end{aligned}
$$

may be enlarged as $\left(\exp \left\{\varepsilon d_{2}\right\}-\exp \left\{\varepsilon d_{1}\right\}\right) / \varepsilon$. This will lead conservative results due to the fact that $d(t)$ cannot achieve $d_{1}$ and $d_{2}$ at the same time. While we apply Lemma 7 to these terms, the method by using reciprocally convex lemma [34] can achieve less conservative results. Moreover, for $\varepsilon>$ 0 , the factor $(\dot{d}(t)-1) \exp \{-\varepsilon d(t)\}$ that appeared in the derivative of Lyapunov functional may be directly enlarged as $\mu \exp \left\{-\varepsilon d_{1}\right\}-\exp \left\{-\varepsilon d_{2}\right\}$. In this paper, we enlarge it as $\chi(\varepsilon, \mu)$ to reduce the conservativeness of the obtained criteria. In the literature $[32,36,37]$, this factor is enlarged as $(\mu-$ $1) \exp \left\{-\varepsilon d_{2}\right\}$, which only holds for $\mu<1$.

Remark 13. The proposed Lyapunov functional (28) contains some triple-integral terms, which has not been used in any of the existing literatures in the same context before. Compared with the existing ones, [33] has shown that such a Lyapunov functional type is very effective in the reduction of conservatism. Besides, the information on the lower bound of the delay is sufficiently used in the Lyapunov functional by introducing the terms such as $\int_{t-d_{m}}^{t-d_{1}} \exp \{\varepsilon(s-$ $t)\} x^{T}(s) Q_{4} x(s) d s, \int_{t-d_{2}}^{t-d_{m}} \exp \{\varepsilon(s-t)\} x^{T}(s) Q_{5} x(s) d s$, and $\int_{t-d(t)}^{t-d_{1}} \exp \{\varepsilon(s-t)\} x^{T}(s) Q_{2} x(s) d s$.

Remark 14. It should be also mentioned that the result obtained in Theorem 10 is delay-range-dependent and decay rate-dependent stability condition for (9), which is less conservative than the previous ones and will be verified in Section 4. Although the large number of introduced free weighting matrices may increase the complexity of computation, utilizing the technique of free weighting matrices would reduce the conservativeness. In addition, the given results can be extended to more general systems with neutral delay $\tau(t)$. That is, $\tau_{1} \leq \tau(t) \leq \tau_{2}$. The results can be obtained by using the similar methods.

The information on the delay derivative may not be available in many cases. The following corollary is therefore given, which can be obtained from Theorem 10 by setting $Q_{2}=0$ and $R_{5}=0$.

Corollary 15. For the given finite set $S$ of modes with transition rates matrix, scalars $\alpha, \beta, \gamma, \varepsilon, \tau, d_{1}, d_{2}$, and constant scalar $d_{m}$ satisfying $d_{1}<d_{m}<d_{2}$, the systems described by (9) are exponentially stable with decay rate $\varepsilon / 2$ and $\sigma=\sqrt{\bar{\lambda} / \lambda}$ if the operator $\mathfrak{D}$ is stable, and there exist symmetric positive matrices $P_{i}>0,(i \in S), Q_{j}>0,(j=1,3,4,5), R_{k}>0$, $(k=1,2,3,4), W_{l}>0,(l=1,2,3,4), Y_{m}>0,(m=$ $1,2,3,4), Z_{n}>0,(n=1,2,3,4)$ and matrices $U_{1}, U_{2}, X_{1}$, $X_{2}, X_{3}, X_{4}$ for any scalars $\varepsilon_{1}, \varepsilon_{2}, \varepsilon_{3}$ and any matrices $T_{k}$, $(k=1,2, \ldots, 23)$ with appropriate dimensions, such that linear matrix inequalities (22) and (65) hold:

$$
\begin{array}{ll}
\text { (i) }\left[\begin{array}{ll}
W_{3} & X_{1} \\
X_{1}^{T} & W_{3}
\end{array}\right]>0, \quad\left[\begin{array}{cc}
Y_{3} & X_{2} \\
X_{2}^{T} & Y_{3}
\end{array}\right]>0, \\
\text { (ii) } \widetilde{\Xi}_{i 0}^{\varepsilon}+\Xi_{i 1}^{\varepsilon}<0, \\
\text { (iii) }\left[\begin{array}{ll}
W_{4} & X_{3} \\
X_{3}^{T} & W_{4}
\end{array}\right]>0, \quad\left[\begin{array}{cc}
Y_{4} & X_{4} \\
X_{4}^{T} & Y_{4}
\end{array}\right]>0,
\end{array}
$$

(iv) $\widetilde{\Xi}_{i 0}^{\varepsilon}+\Xi_{i 2}^{\varepsilon}<0$,

where

$$
\begin{aligned}
\widetilde{\Xi}_{i 0}^{\varepsilon}= & \sum_{m=1}^{23} e_{m} \widetilde{G}_{m} e_{m}^{T}+\mathscr{A}(\text { 更) } \\
& -\frac{\varepsilon d_{1}}{\exp \left\{\varepsilon d_{1}\right\}-1}\left(e_{1}-e_{4}\right) Y_{2}\left(e_{1}^{T}-e_{4}^{T}\right) \\
& -\exp \{-\varepsilon \tau\}\left(\tau e_{1}-e_{20}\right) Z_{1}\left(\tau e_{1}^{T}-e_{20}^{T}\right) \\
& -\exp \left\{-\varepsilon d_{1}\right\}\left(d_{1} e_{1}-e_{10}\right) Z_{2}\left(d_{1} e_{1}^{T}-e_{10}^{T}\right)
\end{aligned}
$$




$$
\begin{aligned}
& -\exp \left\{-\varepsilon d_{m}\right\}\left(\varrho_{1} e_{1}-e_{13}\right) Z_{3}\left(\varrho_{1} e_{1}^{T}-e_{13}^{T}\right) \\
& -\exp \left\{-\varepsilon d_{2}\right\}\left(\varrho_{2} e_{1}-e_{14}\right) Z_{4}\left(\varrho_{2} e_{1}^{T}-e_{14}^{T}\right) \\
& -\frac{\varepsilon \tau}{\exp \{\varepsilon \tau\}-1}\left[e_{19} e_{20}-e_{19}\right] \\
& \times\left[\begin{array}{ll}
W_{1} & U_{1} \\
U_{1}^{T} & W_{1}
\end{array}\right]\left[\begin{array}{c}
e_{19}^{T} \\
e_{20}^{T}-e_{19}^{T}
\end{array}\right] \\
& -\frac{\varepsilon \tau}{\exp \{\varepsilon \tau\}-1}\left[\begin{array}{ll}
e_{1}-e_{17} & e_{17}-e_{15}
\end{array}\right] \\
& \times\left[\begin{array}{cc}
Y_{1} & U_{2} \\
U_{2}^{T} & Y_{1}
\end{array}\right]\left[\begin{array}{c}
e_{1}^{T}-e_{17}^{T} \\
e_{17}^{T}-e_{15}^{T}
\end{array}\right]
\end{aligned}
$$

where

$$
\begin{aligned}
& \widetilde{G}_{2}=R_{1}+R_{2}+\tau^{2} Y_{1}+d_{1}^{2} Y_{2}+\varrho_{1}^{2} Y_{3}+\varrho_{2}^{2} Y_{4}+\frac{\tau^{4}}{4} Z_{1} \\
& \quad+\frac{d_{1}^{4}}{4} Z_{2}+\varrho_{3}^{2} Z_{3}+\varrho_{4}^{2} Z_{4}-T_{2}-T_{2}^{T}, \\
& \widetilde{G}_{3}=T_{3} B_{i}+B_{i}^{T} T_{3}^{T}+\varepsilon_{2} \beta^{2} I, \\
& \widetilde{G}_{4}=\exp \left\{-\varepsilon d_{1}\right\}\left(Q_{4}-Q_{3}\right), \\
& \widetilde{G}_{18}=T_{18} C_{i}+C_{i}^{T} T_{18}^{T}+\varepsilon_{3} \gamma^{2} I .
\end{aligned}
$$

Other $\widetilde{G}_{m}=G_{m},(m \neq 2,3,4,18)$, which have been defined previously.

$$
\begin{aligned}
\bar{\lambda}= & \max _{i \in S}\left\{\lambda_{\max }\left(P_{i}\right)\right\} \\
& +\frac{1-\exp \{-\varepsilon \rho\}}{\varepsilon}\left\{\sum_{j=1, j \neq 2}^{5} \lambda_{\max }\left(Q_{j}\right)+\sum_{k=1}^{4} \lambda_{\max }\left(R_{k}\right)\right\} \\
& +\frac{\exp \{-\varepsilon \rho\}+\varepsilon \rho-1}{\varepsilon^{2}}\left\{\sum_{l=1}^{4} \lambda_{\max }\left(W_{l}\right)+\sum_{m=1}^{4} \lambda_{\max }\left(Y_{m}\right)\right\} \\
& +\frac{2-2 \exp \{-\varepsilon \rho\}+\rho^{2} \varepsilon^{2}-2 \varepsilon \rho}{2 \varepsilon^{3}}\left\{\sum_{n=1}^{4} \lambda_{\max }\left(Z_{n}\right)\right\},
\end{aligned}
$$

and the remaining notations are the same as Theorem 10.

In Theorem 10, it is assumed that $\varepsilon \neq 0$. For $\varepsilon=0$, by L'Hospital's rule, the following asymptotic stability criterion can be obtained.

Corollary 16. For the given finite set $S$ of modes with transition rates matrix, scalars $\alpha, \beta, \gamma, \tau, \tau_{d}, d_{1}, d_{2}, \mu$ and constant scalar $d_{m}$ satisfying $d_{1}<d_{m}<d_{2}$, the systems described by (9) are asymptotically stable if the operator $\mathfrak{D}$ is stable, and there exist symmetric positive matrices $P_{i}>0,(i \in S), Q_{j}>0$, $(j=1,2,3,4,5), R_{k}>0,(k=1,2,3,4,5), W_{l}>0,(l=$ $1,2,3,4), Y_{m}>0,(m=1,2,3,4), Z_{n}>0,(n=1,2,3,4)$ and matrices $U_{1}, U_{2}, X_{1}, X_{2}, X_{3}, X_{4}$ for any scalars $\varepsilon_{1}, \varepsilon_{2}$, $\varepsilon_{3}$ and any matrices $T_{k},(k=1,2, \ldots, 23)$ with appropriate dimensions, such that linear matrix inequalities (22) and (69) hold:

$$
\left[\begin{array}{ll}
W_{3} & X_{1} \\
X_{1}^{T} & W_{3}
\end{array}\right]>0, \quad\left[\begin{array}{ll}
Y_{3} & X_{2} \\
X_{2}^{T} & Y_{3}
\end{array}\right]>0,
$$

(ii) $\Xi_{i 0}+\Xi_{i 1}<0$,

$$
\left[\begin{array}{ll}
W_{4} & X_{3} \\
X_{3}^{T} & W_{4}
\end{array}\right]>0, \quad\left[\begin{array}{cc}
Y_{4} & X_{4} \\
X_{4}^{T} & Y_{4}
\end{array}\right]>0
$$

(iv) $\Xi_{i 0}+\Xi_{i 2}<0$,

where

$$
\begin{aligned}
& \Xi_{i 0}=\sum_{m=1}^{23} e_{m} \bar{G}_{m} e_{m}^{T}+\mathscr{A}(\mathbb{\Psi})-\left(e_{1}-e_{4}\right) Y_{2}\left(e_{1}^{T}-e_{4}^{T}\right) \\
& -\left(\tau e_{1}-e_{20}\right) Z_{1}\left(\tau e_{1}^{T}-e_{20}^{T}\right) \\
& -\left(d_{1} e_{1}-e_{10}\right) Z_{2}\left(d_{1} e_{1}^{T}-e_{10}^{T}\right) \\
& -\left(\varrho_{1} e_{1}-e_{13}\right) Z_{3}\left(\varrho_{1} e_{1}^{T}-e_{13}^{T}\right) \\
& -\left(\varrho_{2} e_{1}-e_{14}\right) Z_{4}\left(\varrho_{2} e_{1}^{T}-e_{14}^{T}\right) \\
& -\left[\begin{array}{ll}
e_{19} & e_{20}-e_{19}
\end{array}\right]\left[\begin{array}{cc}
W_{1} & U_{1} \\
U_{1}^{T} & W_{1}
\end{array}\right]\left[\begin{array}{c}
e_{19}^{T} \\
e_{20}^{T}-e_{19}^{T}
\end{array}\right] \\
& -\left[\begin{array}{ll}
e_{1}-e_{17} & e_{17}-e_{15}
\end{array}\right]\left[\begin{array}{cc}
Y_{1} & U_{2} \\
U_{2}^{T} & Y_{1}
\end{array}\right]\left[\begin{array}{c}
e_{1}^{T}-e_{17}^{T} \\
e_{17}^{T}-e_{15}^{T}
\end{array}\right] \text {, } \\
& \bar{G}_{1}=A_{i}^{T} P_{i}+P_{i} A_{i}+\sum_{j=1}^{N} \pi_{i j} P_{j}+Q_{1}+Q_{3}+\tau^{2} W_{1} \\
& +d_{1}^{2} W_{2}+\varrho_{1}^{2} W_{3}+\varrho_{2}^{2} W_{4}+\varepsilon_{1} \alpha^{2} I+T_{1} A_{i}+A_{i}^{T} T_{1}^{T}, \\
& \bar{G}_{3}=(\mu-1) Q_{2}+T_{3} B_{i}+B_{i}^{T} T_{3}^{T}+\varepsilon_{2} \beta^{2} I, \\
& \bar{G}_{4}=Q_{2}+Q_{4}-Q_{3} \text {, } \\
& \bar{G}_{5}=Q_{5}-Q_{4}, \quad \bar{G}_{6}=-Q_{5}, \\
& \bar{G}_{7}=R_{3}-R_{2}, \quad \bar{G}_{8}=R_{4}-R_{3}, \\
& \bar{G}_{9}=-R_{4}, \quad \bar{G}_{10}=-W_{2}, \\
& \bar{G}_{15}=-Q_{1}, \quad \bar{G}_{16}=-R_{1}, \\
& \bar{G}_{18}=\left(\tau_{d}-1\right) R_{5}+T_{18} C_{i}+C_{i}^{T} T_{18}^{T}+\varepsilon_{3} \gamma^{2} I .
\end{aligned}
$$


Other $\bar{G}_{m}=G_{m},(m=2,11,12,13,14,17,19, \ldots, 23)$, which have been defined in Theorem 10:

$$
\begin{aligned}
\Xi_{i 1}= & -e_{14} W_{4} e_{14}^{T}-\left(e_{5}-e_{6}\right) Y_{4}\left(e_{5}^{T}-e_{6}^{T}\right) \\
& -\left[\begin{array}{ll}
e_{11} & e_{13}-e_{11}
\end{array}\right]\left[\begin{array}{ll}
W_{3} & X_{1} \\
X_{1}^{T} & W_{3}
\end{array}\right]\left[\begin{array}{c}
e_{11}^{T} \\
e_{13}^{T}-e_{11}^{T}
\end{array}\right] \\
& -\left[e_{4}-e_{3} e_{3}-e_{5}\right]\left[\begin{array}{cc}
Y_{3} & X_{2} \\
X_{2}^{T} & Y_{3}
\end{array}\right]\left[\begin{array}{c}
e_{4}^{T}-e_{3}^{T} \\
e_{3}^{T}-e_{5}^{T}
\end{array}\right], \\
\Xi_{i 2}= & -e_{13} W_{3} e_{13}^{T}-\left(e_{4}-e_{5}\right) Y_{3}\left(e_{4}^{T}-e_{5}^{T}\right) \\
& -\left[\begin{array}{lll}
e_{12} & e_{14}-e_{12}
\end{array}\right]\left[\begin{array}{ll}
W_{4} & X_{3} \\
X_{3}^{T} & W_{4}
\end{array}\right]\left[\begin{array}{c}
e_{12}^{T} \\
e_{14}^{T}-e_{12}^{T}
\end{array}\right] \\
& -\left[\begin{array}{ll}
e_{3}-e_{6} & e_{5}-e_{3}
\end{array}\right]\left[\begin{array}{cc}
Y_{4} & X_{4} \\
X_{4}^{T} & Y_{4}
\end{array}\right]\left[\begin{array}{c}
e_{3}^{T}-e_{6}^{T} \\
e_{5}^{T}-e_{3}^{T}
\end{array}\right],
\end{aligned}
$$

and the remaining notations are the same as Theorem 10.

3.2. Exponential Stability for the Nominal Systems with Partially Unknown Transition Rates. In this subsection, we take into account the situation that the information of transition rates is not accessible completely and propose the following conditions to guarantee the exponential stability of system (9) with partially unknown transition rates.

Since the transition rates of the Markov chain are partially unknown, then some elements in matrix $\Pi=\left[\pi_{i j}\right]_{N \times N}$ are inaccessible. For instance, the system (3) with five operation modes, the jump rates matrix $\Pi$ may be viewed as

$$
\left[\begin{array}{ccccc}
? & \pi_{12} & ? & ? & \pi_{15} \\
\pi_{21} & ? & ? & \pi_{24} & ? \\
? & ? & \pi_{33} & \pi_{34} & ? \\
? & \pi_{42} & ? & ? & ? \\
\pi_{51} & ? & ? & ? & \pi_{55}
\end{array}\right]
$$

where? represents the unknown element. For notation clarity, we denote that $\mathcal{S}^{i}=\mathcal{S}_{k}^{i} \cup \mathcal{S}_{u k}^{i}$, for all $i \in S$ and

$$
\begin{gathered}
\mathcal{S}_{k}^{i} \triangleq\left\{j: \pi_{i j} \text { is known for } j \in S\right\}, \\
\mathcal{S}_{u k}^{i} \triangleq\left\{j: \pi_{i j} \text { is unknown for } j \in S\right\} .
\end{gathered}
$$

If $\mathcal{S}_{k}^{i} \neq \emptyset$, it is further described as

$$
\mathcal{S}_{k}^{i}=\left\{k_{1}^{i}, k_{2}^{i}, \ldots, k_{m}^{i}\right\}, \quad 1 \leq m \leq N
$$

where $k_{j}^{i},(j=1,2, \ldots, m)$ represent the $j$ th known element of the set $\mathcal{S}_{k}^{i}$ in the $i$ th row of the transition rate matrix $\Pi$. Specially, when $\mathcal{S}_{u k}^{i}=\emptyset, \mathcal{S}^{i}=\mathcal{S}_{k}^{i}$, the full information about $\Pi$ is obtained, and it becomes the case of Section 3.1.
Theorem 17. For the given finite set $S$ of modes with partly unknown transition rates matrix, scalars $\alpha, \beta, \gamma, \varepsilon, \tau, \tau_{d}, d_{1}$, $d_{2}$, and $\mu$, and constant scalar $d_{m}$ satisfying $d_{1}<d_{m}<d_{2}$, the systems described by (9) with partly unknown transition rates are exponentially stable with decay rate $\varepsilon / 2$ and $\sigma=\sqrt{\bar{\lambda}} / \underline{\lambda}$ if the operator $\mathfrak{D}$ is stable, and there exist symmetric positive matrices $P_{i}>0,(i \in S), Q_{j}>0,(j=1,2,3,4,5), R_{k}>0$, $(k=1,2,3,4,5), W_{l}>0,(l=1,2,3,4), Y_{m}>0,(m=$ $1,2,3,4), Z_{n}>0,(n=1,2,3,4)$ and matrices $U_{1}, U_{2}, X_{1}, X_{2}$, $X_{3}, X_{4}$ for any scalars $\varepsilon_{1}, \varepsilon_{2}, \varepsilon_{3}$ and any symmetric matrices $V_{i}$, $(i \in S)$ and any matrices $T_{k},(k=1,2, \ldots, 23)$ with appropriate dimensions, such that linear matrix inequalities (22), (75), (76), and (77) hold:

$$
\begin{aligned}
& P_{j}-V_{i} \leq 0, \quad j \in \mathcal{S}_{u k}^{i}, j \neq i, \\
& P_{j}-V_{i} \geq 0, \quad j \in \mathcal{S}_{u k}^{i}, j=i, \\
& \text { (i) } \quad\left[\begin{array}{ll}
W_{3} & X_{1} \\
X_{1}^{T} & W_{3}
\end{array}\right]>0, \quad\left[\begin{array}{cc}
Y_{3} & X_{2} \\
X_{2}^{T} & Y_{3}
\end{array}\right]>0,
\end{aligned}
$$

(ii) $\Xi_{\delta i 0}^{\varepsilon}+\Xi_{\delta i 1}^{\varepsilon}<0$,

(iii) $\left[\begin{array}{ll}W_{4} & X_{3} \\ X_{3}^{T} & W_{4}\end{array}\right]>0, \quad\left[\begin{array}{cc}Y_{4} & X_{4} \\ X_{4}^{T} & Y_{4}\end{array}\right]>0$,

(iv) $\Xi_{\delta i 0}^{\varepsilon}+\Xi_{\delta i 2}^{\varepsilon}<0$,

where

$$
\begin{aligned}
& \Xi_{S i 0}^{\varepsilon}=\sum_{m=1}^{23} e_{m} G_{\delta m} e_{m}^{T}+\mathscr{A}(\Psi) \\
& -\frac{\varepsilon d_{1}}{\exp \left\{\varepsilon d_{1}\right\}-1}\left(e_{1}-e_{4}\right) Y_{2}\left(e_{1}^{T}-e_{4}^{T}\right) \\
& -\exp \{-\varepsilon \tau\}\left(\tau e_{1}-e_{20}\right) Z_{1}\left(\tau e_{1}^{T}-e_{20}^{T}\right) \\
& -\exp \left\{-\varepsilon d_{1}\right\}\left(d_{1} e_{1}-e_{10}\right) Z_{2}\left(d_{1} e_{1}^{T}-e_{10}^{T}\right) \\
& -\exp \left\{-\varepsilon d_{m}\right\}\left(\varrho_{1} e_{1}-e_{13}\right) Z_{3}\left(\varrho_{1} e_{1}^{T}-e_{13}^{T}\right) \\
& -\exp \left\{-\varepsilon d_{2}\right\}\left(\varrho_{2} e_{1}-e_{14}\right) Z_{4}\left(\varrho_{2} e_{1}^{T}-e_{14}^{T}\right) \\
& -\frac{\varepsilon \tau}{\exp \{\varepsilon \tau\}-1}\left[\begin{array}{ll}
e_{19} & e_{20}-e_{19}
\end{array}\right] \\
& \times\left[\begin{array}{cc}
W_{1} & U_{1} \\
U_{1}^{T} & W_{1}
\end{array}\right]\left[\begin{array}{c}
e_{19}^{T} \\
e_{20}^{T}-e_{19}^{T}
\end{array}\right] \\
& -\frac{\varepsilon \tau}{\exp \{\varepsilon \tau\}-1}\left[e_{1}-e_{17} e_{17}-e_{15}\right] \\
& \times\left[\begin{array}{cc}
Y_{1} & U_{2} \\
U_{2}^{T} & Y_{1}
\end{array}\right]\left[\begin{array}{c}
e_{1}^{T}-e_{17}^{T} \\
e_{17}^{T}-e_{15}^{T}
\end{array}\right],
\end{aligned}
$$




$$
\begin{aligned}
G_{\delta 1}= & A_{i}^{T} P_{i}+P_{i} A_{i}+\sum_{j \in \delta_{k}^{i}}^{N} \pi_{i j}\left(P_{j}-V_{i}\right) \\
& +\varepsilon P_{i}+Q_{1}+Q_{3}+\tau^{2} W_{1}+d_{1}^{2} W_{2}+\varrho_{1}^{2} W_{3} \\
& +\varrho_{2}^{2} W_{4}+\varepsilon_{1} \alpha^{2} I+T_{1} A_{i}+A_{i}^{T} T_{1}^{T}, \\
G_{\delta m}= & G_{m}, \quad(m \neq 1), \quad \Xi_{\delta i 1}^{\varepsilon}=\Xi_{i 1}^{\varepsilon}, \quad \Xi_{\delta i 2}^{\varepsilon}=\Xi_{i 2}^{\varepsilon},
\end{aligned}
$$

and the remaining notations are the same as Theorem 10.

Proof. Choose the same Lyapunov functional as (28), and $V_{2}\left(x_{t}, i\right), V_{3}\left(x_{t}, i\right), V_{4}\left(x_{t}, i\right), V_{5}\left(x_{t}, i\right)$, and $V_{6}\left(x_{t}, i\right)$ are disposed with the identical method in Theorem 10. Then, giving consideration on $\sum_{j=1}^{N} \pi_{i j}=0$, the following equation holds for arbitrary matrices $V_{i}=V_{i}^{T}, i \in S$; that is,

$$
-x^{T}(t)\left(\sum_{j=1}^{N} \pi_{i j} V_{i}\right) x(t)=0 .
$$

With (36) and (79), we obtain the following:

$$
\begin{aligned}
\mathfrak{L} V_{1}\left(x_{t}, i\right)=2[ & x^{T}(t) A_{i}^{T}+x^{T}(t-d(t)) B_{i}^{T} \\
& \left.+\dot{x}^{T}(t-\tau(t)) C_{i}^{T}+f_{1}^{T}+f_{2}^{T}+f_{3}^{T}\right] P_{i} x(t) \\
& +x^{T}(t)\left[\sum_{j \in \mathcal{S}_{k}^{i}}^{N} \pi_{i j}\left(P_{j}-V_{i}\right)\right] x(t) \\
& \left.+x^{T}(t)\left[\sum_{j \in \mathcal{S}_{u k}^{i}}^{N} \pi_{i j}\left(P_{j}-V_{i}\right)\right] x(t)\right] \\
& +\varepsilon x^{T}(t) P_{i} x(t)-\varepsilon V_{1}\left(x_{t}, i\right) .
\end{aligned}
$$

For $d(t) \in\left[d_{1}, d_{m}\right]$ and $d(t) \in\left[d_{m}, d_{2}\right]$, with (80) and on the basis of the result in Theorem 10, we have the following equations, respectively:

$$
\begin{aligned}
& \mathfrak{Q V}(x(t), i, t)+\varepsilon V(x(t), i, t) \\
& \leq \xi^{T}(t)\left(\Xi_{\delta i 0}^{\varepsilon}+\Xi_{\delta i 1}^{\varepsilon}\right) \xi(t) \\
& \quad+x^{T}(t)\left[\sum_{j \in \delta_{u k}^{i}}^{N} \pi_{i j}\left(P_{j}-V_{i}\right)\right] x(t), \\
& \mathfrak{Q} V(x(t), i, t)+\varepsilon V(x(t), i, t) \\
& \leq \xi^{T}(t)\left(\Xi_{\delta i 0}^{\varepsilon}+\Xi_{\delta i 2}^{\varepsilon}\right) \xi(t) \\
& \quad+x^{T}(t)\left[\sum_{j \in \delta_{u k}^{i}}^{N} \pi_{i j}\left(P_{j}-V_{i}\right)\right] x(t) .
\end{aligned}
$$

With (81), due to $\lambda_{i i}=-\sum_{j=1, j \neq i}^{N} \lambda_{i j}$ and $\lambda_{i j}>0$, for all $i \neq j$, (59) is also held for $d(t) \in\left[d_{1}, d_{2}\right]$ if linear matrix inequalities (22), (75), (76), and (77) are satisfied. With the same procedure in the latter proof of Theorem 10, we draw the conclusion that the system (9) with partially unknown transition rates is exponentially stable with a decay rate $\varepsilon / 2$. This completes the proof.

Consider the system (9) with partially unknown transition rates, and the following corollaries are given, for unknown information on the delay derivative and $\varepsilon=0$, respectively.

Corollary 18. For the given finite set $S$ of modes with partly unknown transition rates matrix, scalars $\alpha, \beta, \gamma, \varepsilon, \tau, d_{1}$, and $d_{2}$, and constant scalar $d_{m}$ satisfying $d_{1}<d_{m}<d_{2}$, the systems described by (9) with partly unknown transition rates are exponentially stable with decay rate $\varepsilon / 2$ and $\sigma=\sqrt{\bar{\lambda} / \lambda}$ if the operator $\mathfrak{D}$ is stable, and there exist symmetric positive matrices $P_{i}>0,(i \in S), Q_{j}>0,(j=1,3,4,5), R_{k}>0$, $(k=1,2,3,4), W_{l}>0,(l=1,2,3,4), Y_{m}>0,(m=1,2,3,4)$, $Z_{n}>0,(n=1,2,3,4)$ and matrices $U_{1}, U_{2}, X_{1}, X_{2}, X_{3}$, $X_{4}$ for any scalars $\varepsilon_{1}, \varepsilon_{2}, \varepsilon_{3}$ and any symmetric matrices $V_{i}$, $(i \in S)$ and any matrices $T_{k},(k=1,2, \ldots, 23)$ with appropriate dimensions, such that linear matrix inequalities (22), (75), and (82) hold:

$$
\begin{array}{ll}
\text { (i) } & {\left[\begin{array}{ll}
W_{3} & X_{1} \\
X_{1}^{T} & W_{3}
\end{array}\right]>0, \quad\left[\begin{array}{cc}
Y_{3} & X_{2} \\
X_{2}^{T} & Y_{3}
\end{array}\right]>0,} \\
\text { (ii) } \widetilde{\Xi}_{\delta i 0}^{\varepsilon}+\Xi_{s i 1}^{\varepsilon}<0, & {\left[\begin{array}{ll}
W_{4} & X_{3} \\
X_{3}^{T} & W_{4}
\end{array}\right]>0, \quad\left[\begin{array}{cc}
Y_{4} & X_{4} \\
X_{4}^{T} & Y_{4}
\end{array}\right]>0,}
\end{array}
$$

(iv) $\widetilde{\Xi}_{\delta i 0}^{\varepsilon}+\Xi_{\delta i 2}^{\varepsilon}<0$,

where

$$
\begin{aligned}
\widetilde{\Xi}_{\delta i 0}^{\varepsilon}= & \sum_{m=1}^{23} e_{m} \widetilde{G}_{\delta m} e_{m}^{T}+\mathscr{A}(\text { 出 }) \\
& -\frac{\varepsilon d_{1}}{\exp \left\{\varepsilon d_{1}\right\}-1}\left(e_{1}-e_{4}\right) Y_{2}\left(e_{1}^{T}-e_{4}^{T}\right) \\
& -\exp \{-\varepsilon \tau\}\left(\tau e_{1}-e_{20}\right) Z_{1}\left(\tau e_{1}^{T}-e_{20}^{T}\right) \\
& -\exp \left\{-\varepsilon d_{1}\right\}\left(d_{1} e_{1}-e_{10}\right) Z_{2}\left(d_{1} e_{1}^{T}-e_{10}^{T}\right) \\
& -\exp \left\{-\varepsilon d_{m}\right\}\left(\varrho_{1} e_{1}-e_{13}\right) Z_{3}\left(\varrho_{1} e_{1}^{T}-e_{13}^{T}\right) \\
& -\exp \left\{-\varepsilon d_{2}\right\}\left(\varrho_{2} e_{1}-e_{14}\right) Z_{4}\left(\varrho_{2} e_{1}^{T}-e_{14}^{T}\right) \\
& -\frac{\varepsilon \tau}{\exp \{\varepsilon \tau\}-1}\left[e_{19} e_{20}-e_{19}\right] \\
& \times\left[\begin{array}{ll}
W_{1} & U_{1} \\
U_{1}^{T} & W_{1}
\end{array}\right]\left[\begin{array}{c}
e_{19}^{T} \\
e_{20}^{T}-e_{19}^{T}
\end{array}\right]
\end{aligned}
$$




$$
\begin{aligned}
& -\frac{\varepsilon \tau}{\exp \{\varepsilon \tau\}-1}\left[\begin{array}{ll}
e_{1}-e_{17} & e_{17}-e_{15}
\end{array}\right] \\
& \times\left[\begin{array}{cc}
Y_{1} & U_{2} \\
U_{2}^{T} & Y_{1}
\end{array}\right]\left[\begin{array}{c}
e_{1}^{T}-e_{17}^{T} \\
e_{17}^{T}-e_{15}^{T}
\end{array}\right], \\
\widetilde{G}_{\mathcal{S} 1}= & G_{\mathcal{S} 1}, \quad \widetilde{G}_{\delta m}=\widetilde{G}_{m}, \quad(m \neq 1), \\
\Xi_{\delta i 1}^{\varepsilon}= & \Xi_{i 1}^{\varepsilon}, \quad \Xi_{\delta i 2}^{\varepsilon}=\Xi_{i 2}^{\varepsilon},
\end{aligned}
$$

and the remaining notations are the same as Corollary 15.

Corollary 19. For the given finite set $S$ of modes with partly unknown transition rates matrix, scalars $\alpha, \beta, \gamma, \tau, \tau_{d}, d_{1}$, $d_{2}, \mu$, and constant scalar $d_{m}$ satisfying $d_{1}<d_{m}<d_{2}$, the systems described by (9) with partly unknown transition rates are exponentially stable with decay rate $\varepsilon / 2$ and $\sigma=\sqrt{\bar{\lambda} / \underline{\lambda}}$ if the operator $\mathfrak{D}$ is stable, and there exist symmetric positive matrices $P_{i}>0,(i \in S), Q_{j}>0,(j=1,2,3,4,5), R_{k}>0$, $(k=1,2,3,4,5), W_{l}>0,(l=1,2,3,4), Y_{m}>0,(m=$ $1,2,3,4), Z_{n}>0,(n=1,2,3,4)$ and matrices $U_{1}, U_{2}, X_{1}, X_{2}$, $X_{3}, X_{4}$ for any scalars $\varepsilon_{1}, \varepsilon_{2}, \varepsilon_{3}$ and any symmetric matrices $V_{i}$, $(i \in S)$ and any matrices $T_{k},(k=1,2, \ldots, 23)$ with appropriate dimensions, such that linear matrix inequalities (22), (75), and (84) hold:
(i) $\left[\begin{array}{ll}W_{3} & X_{1} \\ X_{1}^{T} & W_{3}\end{array}\right]>0, \quad\left[\begin{array}{cc}Y_{3} & X_{2} \\ X_{2}^{T} & Y_{3}\end{array}\right]>0$,
(ii) $\Xi_{\delta i 0}+\Xi_{\delta i 1}<0$,
(iii) $\left[\begin{array}{ll}W_{4} & X_{3} \\ X_{3}^{T} & W_{4}\end{array}\right]>0, \quad\left[\begin{array}{cc}Y_{4} & X_{4} \\ X_{4}^{T} & Y_{4}\end{array}\right]>0$,
(iv) $\Xi_{\delta i 0}+\Xi_{\delta i 2}<0$

where

$$
\begin{aligned}
\Xi_{\delta i 0}= & \sum_{m=1}^{23} e_{m} \bar{G}_{\delta m} e_{m}^{T}+\mathscr{A}(\Psi) \\
& -\left(e_{1}-e_{4}\right) Y_{2}\left(e_{1}^{T}-e_{4}^{T}\right) \\
& -\left(\tau e_{1}-e_{20}\right) Z_{1}\left(\tau e_{1}^{T}-e_{20}^{T}\right) \\
& -\left(d_{1} e_{1}-e_{10}\right) Z_{2}\left(d_{1} e_{1}^{T}-e_{10}^{T}\right) \\
& -\left(\varrho_{1} e_{1}-e_{13}\right) Z_{3}\left(\varrho_{1} e_{1}^{T}-e_{13}^{T}\right) \\
& -\left(\varrho_{2} e_{1}-e_{14}\right) Z_{4}\left(\varrho_{2} e_{1}^{T}-e_{14}^{T}\right) \\
& -\left[e_{19} e_{20}-e_{19}\right]\left[\begin{array}{cc}
W_{1} & U_{1} \\
U_{1}^{T} & W_{1}
\end{array}\right]\left[\begin{array}{c}
e_{19}^{T} \\
e_{20}^{T}-e_{19}^{T}
\end{array}\right]
\end{aligned}
$$

$$
\begin{aligned}
& -\left[\begin{array}{ll}
e_{1}-e_{17} & e_{17}-e_{15}
\end{array}\right] \\
& \times\left[\begin{array}{cc}
Y_{1} & U_{2} \\
U_{2}^{T} & Y_{1}
\end{array}\right]\left[\begin{array}{c}
e_{1}^{T}-e_{17}^{T} \\
e_{17}^{T}-e_{15}^{T}
\end{array}\right] \\
\bar{G}_{\delta 1}= & A_{i}^{T} P_{i}+P_{i} A_{i}+\sum_{j \in \delta_{k}^{i}}^{N} \pi_{i j}\left(P_{j}-V_{i}\right) \\
& +Q_{1}+Q_{3}+\tau^{2} W_{1}+d_{1}^{2} W_{2} \\
& +\varrho_{1}^{2} W_{3}+\varrho_{2}^{2} W_{4}+\varepsilon_{1} \alpha^{2} I+T_{1} A_{i}+A_{i}^{T} T_{1}^{T}, \\
\bar{G}_{\delta m}= & \bar{G}_{m}, \quad(m \neq 1), \quad \Xi_{\delta i 1}=\Xi_{i 1}, \quad \Xi_{\delta i 2}=\Xi_{i 2},
\end{aligned}
$$

and the remaining notations are the same as Corollary 16.

Remark 20. Corollaries 16 and 19 provide new delay-rangedependent asymptotic stability conditions for the systems described by (9) with known and partially unknown transition rates. Though reciprocally convex lemma has not helped here, the results are still less conservative than some previous ones because of some triple-integral terms, which will be verified in Section 4.

3.3. Extension to the Uncertain Case. In this subsection, the uncertain neutral delay Markovian jump systems with nonlinear perturbations described by (3) with known and partially unknown transition rates are, respectively, considered. The corresponding conditions are presented in the following theorems and corollaries.

Theorem 21. For the given finite set $S$ of modes with transition rates matrix, scalars $\alpha, \beta, \gamma, \varepsilon, \tau, \tau_{d}, d_{1}, d_{2}, \mu$, and constant scalar $d_{m}$ satisfying $d_{1}<d_{m}<d_{2}$, the uncertain neutral systems described by (3) are exponentially stable with decay rate $\varepsilon / 2$ and $\sigma=\sqrt{\bar{\lambda}} / \underline{\lambda}$ if the operator $\mathfrak{D}$ is stable, and there exist scalars $\delta_{1}>0, \delta_{2}>0$, symmetric positive matrices $P_{i}>0$, $(i \in S), Q_{j}>0,(j=1,2,3,4,5), R_{k}>0,(k=1,2,3,4,5)$, $W_{l}>0,(l=1,2,3,4), Y_{m}>0,(m=1,2,3,4), Z_{n}>0$, ( $n=1,2,3,4)$, and matrices $U_{1}, U_{2}, X_{1}, X_{2}, X_{3}, X_{4}$ for any scalars $\varepsilon_{1}, \varepsilon_{2}, \varepsilon_{3}$ and any matrices $T_{k},(k=1,2, \ldots, 23)$ with appropriate dimensions, such that (22), (86), and (87) hold:$$
\left[\begin{array}{ll}
W_{3} & X_{1} \\
X_{1}^{T} & W_{3}
\end{array}\right]>0, \quad\left[\begin{array}{cc}
Y_{3} & X_{2} \\
X_{2}^{T} & Y_{3}
\end{array}\right]>0,
$$$$
\text { (ii) }\left[\begin{array}{cc}
\delta_{1} \Xi_{i 0}^{\varepsilon}+\delta_{1} \Xi_{i 1}^{\varepsilon}+\delta_{1}^{2} M^{T} M & N^{T} \\
* & -I
\end{array}\right]<0 \text {, }
$$$$
\text { (iii) }\left[\begin{array}{ll}
W_{4} & X_{3} \\
X_{3}^{T} & W_{4}
\end{array}\right]>0, \quad\left[\begin{array}{cc}
Y_{4} & X_{4} \\
X_{4}^{T} & Y_{4}
\end{array}\right]>0 \text {, }
$$$$
\text { (iv) }\left[\begin{array}{cc}
\delta_{2} \Xi_{i 0}^{\varepsilon}+\delta_{2} \Xi_{i 2}^{\varepsilon}+\delta_{2}^{2} M^{T} M & N^{T} \\
* & -I
\end{array}\right]<0 \text {, }
$$ 
where

$$
M=e_{1} E_{A i}^{T}+e_{3} E_{B i}^{T}, \quad N=H_{i}^{T} P_{i}^{T} e_{1}+\sum_{m=1}^{23} H_{i}^{T} T_{m}^{T} e_{m}
$$

and other notations are the same as Theorem 10.

Proof. Define $E=\operatorname{col}\left\{e_{m},(m=1,2, \ldots, 23)\right\}$ and $\widehat{T}=$ $\operatorname{col}\left\{P_{i}+T_{1}, T_{m}(m=2, \ldots, 23)\right\}$, and we replace $A_{i}, B_{i}$ with $A_{i}+\Delta A_{i}(t), B_{i}+\Delta B_{i}(t)$ on the basis of Theorem 10; that is,

(i) $\left[\begin{array}{ll}W_{3} & X_{1} \\ X_{1}^{T} & W_{3}\end{array}\right]>0, \quad\left[\begin{array}{cc}Y_{3} & X_{2} \\ X_{2}^{T} & Y_{3}\end{array}\right]>0$,
(ii) $\Xi_{i 0}^{\varepsilon}+\Xi_{i 1}^{\varepsilon}+\mathscr{A}\left[e_{1} \Delta A_{i}^{T}(t) \widehat{T}^{T} E+e_{3} \Delta B_{i}^{T}(t) \widehat{T}^{T} E\right]<0$,

(iii) $\left[\begin{array}{ll}W_{4} & X_{3} \\ X_{3}^{T} & W_{4}\end{array}\right]>0, \quad\left[\begin{array}{cc}Y_{4} & X_{4} \\ X_{4}^{T} & Y_{4}\end{array}\right]>0$,

(iv) $\Xi_{i 0}^{\varepsilon}+\Xi_{i 2}^{\varepsilon}+\mathscr{A}\left[e_{1} \Delta A_{i}^{T}(t) \widehat{T}^{T} E+e_{3} \Delta B_{i}^{T}(t) \widehat{T}^{T} E\right]<0$.

Considering (ii) of (89) and combining the uncertainties condition (7), we have

$$
\Xi_{i 0}^{\varepsilon}+\Xi_{i 1}^{\varepsilon}+\mathscr{A}\left\{\left[\begin{array}{ll}
e_{1} & e_{3}
\end{array}\right]\left[\begin{array}{c}
E_{A i}^{T} \\
E_{B i}^{T}
\end{array}\right] F_{i}^{T}(t) H_{i}^{T} \widehat{T}^{T} E\right\}<0
$$

By the definition of $\mathscr{A}$, we obtain

$$
\Xi_{i 0}^{\varepsilon}+\Xi_{i 1}^{\varepsilon}+M F_{i}^{T}(t) N+N^{T} F_{i}(t) M^{T}<0
$$

According to (8), by Lemmas 9 and 6, with (92), we obtain (ii) of (86). Following the same procedure, (90) is considered and (iv) of (87) can be obtained. Finally, following the proof of Theorem 10, the systems described by (3) are exponentially stable with a decay rate $\varepsilon / 2$. This completes the proof.

Theorem 22. For the given finite set $S$ of modes with partly unknown transition rates matrix, scalars $\alpha, \beta, \gamma, \varepsilon, \tau, \tau_{d}, d_{1}$, $d_{2}, \mu$, and constant scalar $d_{m}$ satisfying $d_{1}<d_{m}<d_{2}$, the uncertain neutral systems described by (3) with partly unknown transition rates are exponentially stable with decay rate $\varepsilon / 2$ and $\sigma=\sqrt{\bar{\lambda} / \lambda}$ if the operator $\mathfrak{D}$ is stable, and there exist scalars $\delta_{1}>0, \delta_{2}>0$, symmetric positive matrices $P_{i}>0$, $(i \in S), Q_{j}>0,(j=1,2,3,4,5), R_{k}>0,(k=1,2,3,4,5)$, $W_{l}>0,(l=1,2,3,4), Y_{m}>0,(m=1,2,3,4), Z_{n}>0$, $(n=1,2,3,4)$, and matrices $U_{1}, U_{2}, X_{1}, X_{2}, X_{3}, X_{4}$ for any scalars $\varepsilon_{1}, \varepsilon_{2}, \varepsilon_{3}$ and any symmetric matrices $V_{i},(i \in S)$ and any matrices $T_{k},(k=1,2, \ldots, 23)$ with appropriate dimensions, such that (22), (75), and (93) hold:

$$
\begin{aligned}
& \text { (i) }\left[\begin{array}{ll}
W_{3} & X_{1} \\
X_{1}^{T} & W_{3}
\end{array}\right]>0, \quad\left[\begin{array}{cc}
Y_{3} & X_{2} \\
X_{2}^{T} & Y_{3}
\end{array}\right]>0 \text {, } \\
& \text { (ii) }\left[\begin{array}{cc}
\delta_{1} \Xi_{\mathscr{I} i 0}^{\varepsilon}+\delta_{1} \Xi_{\mathscr{f} i 1}^{\varepsilon}+\delta_{1}^{2} M^{T} M & N^{T} \\
* & -I
\end{array}\right]<0 \text {, } \\
& \text { (iii) }\left[\begin{array}{ll}
W_{4} & X_{3} \\
X_{3}^{T} & W_{4}
\end{array}\right]>0, \quad\left[\begin{array}{cc}
Y_{4} & X_{4} \\
X_{4}^{T} & Y_{4}
\end{array}\right]>0 \text {, } \\
& \text { (iv) }\left[\begin{array}{cc}
\delta_{2} \Xi_{\mathscr{I} i 0}^{\varepsilon}+\delta_{2} \Xi_{\mathscr{I}_{i 2}}^{\varepsilon}+\delta_{2}^{2} M^{T} M & N^{T} \\
* & -I
\end{array}\right]<0 \text {, }
\end{aligned}
$$

where

$$
M=e_{1} E_{A i}^{T}+e_{3} E_{B i}^{T}, \quad N=H_{i}^{T} P_{i}^{T} e_{1}+\sum_{m=1}^{23} H_{i}^{T} T_{m}^{T} e_{m}
$$

and other notations are the same as Theorem 17.

The proof of Theorem 22 is omitted here because it is identical with the proof of Theorem 21. In addition, considering the system (3) with fully or partly known transition rates, the corollaries for unknown information on the delay derivative and $\varepsilon=0$ are also omitted here because they are uniform results as the nominal case.

\section{Numerical Examples}

In this section, numerical examples are provided with known and partially unknown transition rates, respectively, which demonstrate that the proposed theoretical results in this paper are effectiveness.

4.1. Systems with Known Transition Rates. In this subsection, numerical systems with full information on transition rates are given to show the effectiveness of ours.

Example 1. Partial element equivalent circuit (PEEC) model can be represented as a stochastic jump system as in (3) with the abrupt variation in structures and parameters [38]. The practical system is given as follows:

$$
\begin{aligned}
\dot{x}(t) & -C_{i} \dot{x}(t-\tau(t)) \\
= & \left(A_{i}+\Delta A(t)\right) x(t)+\left(B_{i}+\Delta B(t)\right) x(t-d(t)),
\end{aligned}
$$


TABLE 1: Maximum exponential decay rate $\widehat{\varepsilon}$ with different parameter $d$.

\begin{tabular}{lcccccccc}
\hline Methods & $d$ & 0.8 & 1.0 & 1.2 & 1.4 & 1.6 & 1.8 & 2.0 \\
\hline Mondie and Kharitonov [39] & $\widehat{\varepsilon}$ & 0.7344 & 0.6715 & 0.6145 & 0.5642 & 0.5202 & 0.4818 & 0.4481 \\
Liu [40] & $\widehat{\varepsilon}$ & 0.9367 & 0.5903 & 0.3400 & 0.1813 & 0.0752 & 0.0014 & 0 \\
Xu et al. [41] & $\widehat{\varepsilon}$ & 0.9366 & 0.9192 & 0.8991 & 0.8115 & 0.6990 & 0.6148 & 0.5494 \\
Theorem 10 & $\widehat{\varepsilon}$ & 0.9569 & 0.9407 & 0.9218 & 0.9024 & 0.8154 & 0.7394 & 0.6933 \\
\hline
\end{tabular}

TABLE 2: Maximum exponential decay rate $\widehat{\varepsilon}$ with different parameter $d$.

\begin{tabular}{lcccccccc}
\hline Methods & $d$ & 0.3 & 0.5 & 0.7 & 0.9 & 1.1 & 1.3 & 1.5 \\
\hline Mondie and Kharitonov [39] & $\widehat{\varepsilon}$ & 0.6255 & 0.4760 & 0.3825 & 0.3191 & 0.2735 & 0.2392 & 0.2125 \\
Xu et al. [41] & $\widehat{\varepsilon}$ & 1.0108 & 0.8366 & 0.7103 & 0.6156 & 0.5425 & 0.4845 & 0.4375 \\
Theorem 21 & $\widehat{\varepsilon}$ & 1.0419 & 0.9207 & 0.8035 & 0.7048 & 0.6234 & 0.5876 & 0.5043 \\
\hline
\end{tabular}

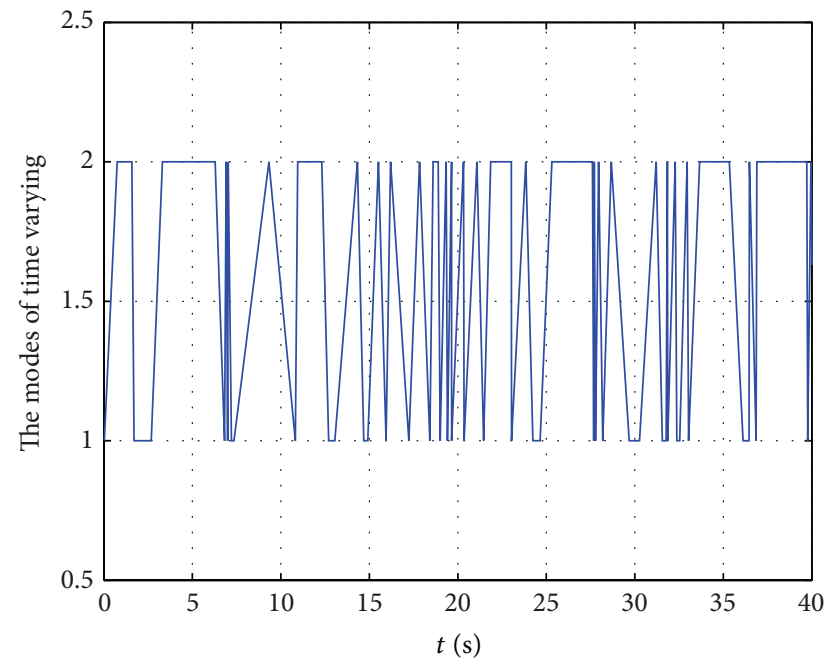

FIgURE 1: The mode switching of Example 1.

where $\alpha=\beta=\gamma=0, i \in S=\{1,2\}$, and the mode switching is governed by the rate matrix $\Pi=\left[\begin{array}{cc}-3 & 3 \\ 2 & -2\end{array}\right]$ as shown in Figure 1, and the state matrices

$$
\begin{array}{cc}
A_{1}=\left[\begin{array}{cc}
-4 & 0 \\
0 & -3
\end{array}\right], & A_{2}=\left[\begin{array}{cc}
-2 & 0 \\
0 & -3
\end{array}\right], \\
B_{1}=\left[\begin{array}{cc}
-1 & 0 \\
-1 & -1
\end{array}\right], & B_{2}=\left[\begin{array}{cc}
-1 & 0 \\
-0.9 & -1.2
\end{array}\right], \\
C_{1}=0.5 I, & C_{2}=0.3 I, \\
H_{1}=\left[\begin{array}{l}
0.2 \\
0.2
\end{array}\right], & H_{2}=\left[\begin{array}{c}
0 \\
-0.3
\end{array}\right], \\
E_{A 1}=\left[\begin{array}{ll}
0.2 & 0
\end{array}\right], & E_{A 2}=\left[\begin{array}{ll}
0 & 0.2
\end{array}\right], \\
E_{B 1}=\left[\begin{array}{ll}
-0.3 & 0.3
\end{array}\right], & E_{B 2}=\left[\begin{array}{ll}
0.2 & 0.2
\end{array}\right] .
\end{array}
$$

Given the decay rate $\varepsilon / 2=0.4$, the time-varying neutral delay $\tau(t)=0.1 \sin ^{2} t$, and retarded delay $d(t)=0.1\left(1+\cos ^{2} t\right)$, from $\tau(t), d(t)$ with $t \in[0,2 \pi]$ in Figures 2 and 3 , it is readily obtained that $\tau=0.1, d_{1}=0.1$, and $d_{2}=0.2$. Without loss of generality, we choose $d_{m}=0.12$. In addition, we have

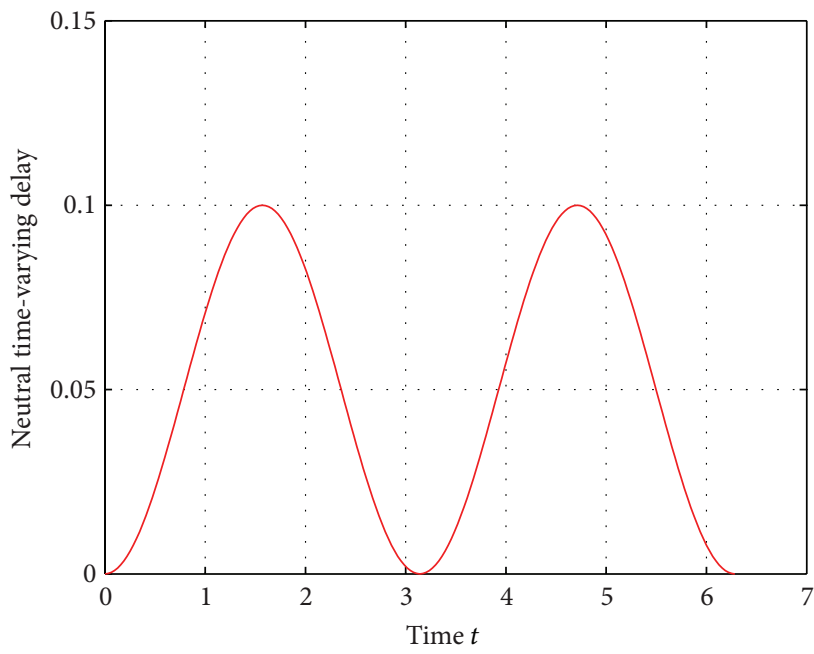

Figure 2: Interval time-varying neutral delay $\tau(t)$ of Example 1.

$\dot{\tau}(t)=0.1 \sin 2 t, \tau_{d}=0.1$ and $\dot{d}(t)=-0.1 \sin 2 t, \mu=0.1$. Thus, the exponential stability can be readily established by Theorem 21.

Example 2. To show the reduced conservativeness of the exponential stability condition in Theorem 10 , consider the time-delay system in the form of (9) with $C_{i}=0, \alpha=\beta=$ $\gamma=0, d(t)=d$, and

$$
A_{i}=\left[\begin{array}{cc}
-3 & -2 \\
1 & 0
\end{array}\right], \quad B_{i}=\left[\begin{array}{cc}
-0.5 & 0.1 \\
0.3 & 0
\end{array}\right], \quad i \in S=\{1\} .
$$

For given $d$, by Theorem 10, the maximum exponential decay rate $\widehat{\varepsilon}$, which satisfies the LMIs in (22), (23), and (24), can be calculated by solving a quasi-convex optimization problem. The results are presented in Table 1.

From Table 1, we know that the maximum exponential decay rate $\widehat{\varepsilon}=0.9569$ in this paper by setting $d=0.8$, while the maximum exponential decay rate $\widehat{\varepsilon}=0.7344$ for [39], $\widehat{\varepsilon}=$ 0.9367 for [40], and $\widehat{\varepsilon}=0.9366$ for [41]. The results are also given by setting $d=1.0, d=1.2, d=1.4, d=1.6, d=1.8$, and $d=2.0$, and it is found that the maximum exponential 
TABLE 3: Maximum upper bound of $d_{2}$ with different $\varepsilon$ and parameter $\mu=0$.

\begin{tabular}{|c|c|c|c|c|c|c|}
\hline Methods & $\widehat{\varepsilon}$ & 0.1 & 0.3 & 0.5 & 0.7 & 0.9 \\
\hline Chen et al. [42] & $d_{2}$ & 1.2999 & 0.8781 & 0.6917 & 0.5792 & 0.5015 \\
\hline Qiu and Cui [10] & $d_{2}$ & 1.4008 & 1.0199 & 0.8457 & 0.7395 & 0.6667 \\
\hline Theorem 10 with $\varepsilon=2 \widehat{\varepsilon}$ & $d_{2}$ & 1.5167 & 1.1365 & 1.0674 & 0.9677 & 0.7558 \\
\hline
\end{tabular}

TABLE 4: Maximum upper bound of $d_{2}$ with different $\varepsilon$ and parameter $\mu=0.5$.

\begin{tabular}{lccccrr}
\hline Methods & $\widehat{\varepsilon}$ & 0.1 & 0.3 & 0.5 & 0.7 & 0.9 \\
\hline Chen et al. [42] & $d_{2}$ & 0.9442 & 0.7275 & 0.6096 & 0.5321 & 0.4761 \\
Qiu and Cui [10] & $d_{2}$ & 1.0120 & 0.8324 & 0.7311 & 0.6941 & 0.6063 \\
Theorem 10 with $\varepsilon=2 \widehat{\varepsilon}$ & $d_{2}$ & 1.0643 & 0.9156 & 0.7985 & 0.7368 & 0.6849 \\
\hline
\end{tabular}

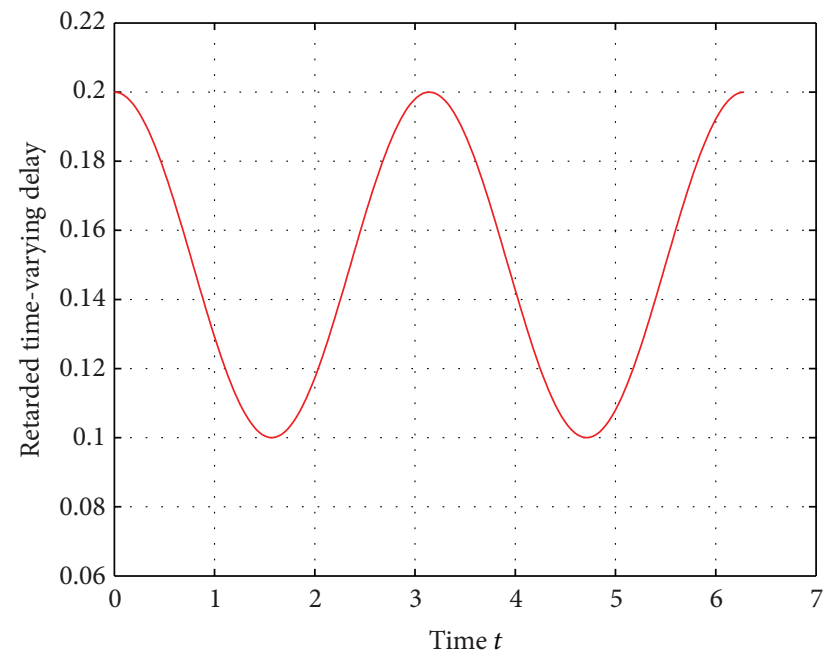

Figure 3: Interval time-varying retarded delay $d(t)$ of Example 1.

decay rate in this paper is larger than those in [39-41]. So, it can be demonstrated that Theorem 10 in this paper yields less conservative results than [39-41].

Consider the previous system again but with parameter uncertainties as follows:

$$
\dot{x}(t)=\left(A_{i}+\Delta A_{i}(t)\right) x(t)+\left(B_{i}+\Delta B_{i}(t)\right) x(t-d),
$$

where

$$
A_{i}=\left[\begin{array}{cc}
-4 & 1 \\
0 & -4
\end{array}\right], \quad B_{i}=\left[\begin{array}{cc}
0.1 & 0 \\
4 & 0.1
\end{array}\right], \quad i \in S=\{1\},
$$

and the uncertain matrices $\Delta A_{i}(t)$ and $\Delta B_{i}(t)$ satisfy

$$
H_{i}=0.2 I, \quad E_{A i}=E_{B i}=I, \quad\left\|F_{i}(t)\right\|<1, \quad i \in S=\{1\} .
$$

For given $d$, by Theorem 21, the maximum exponential decay rate $\widehat{\varepsilon}$, which satisfies the LMIs in (22), (86) and (87), can be calculated by solving a quasi-convex optimization problem. The results are presented in Table 2, where the method of [40] is no longer applicable.

From Table 2, we know that the maximum exponential decay rate $\widehat{\varepsilon}=1.0419$ in this paper by setting $d=0.3$, while the maximum exponential decay rate $\widehat{\varepsilon}=0.6255$ for [39] and $\widehat{\varepsilon}=1.0108$ for [41]. The results are also given by setting $d=0.5, d=0.7, d=0.9, d=1.1, d=1.3$, and $d=1.5$, and it is found that the maximum exponential decay rate in this paper is larger than those in $[3,22,26]$. So, it can be seen that the delay-range-dependent and rate-dependent exponential stability conditions in Theorem 21 in this paper are less conservative than previous results in $[39,41]$.

Example 3. Consider the system (3) with $\tau(t)=d(t)$, and the parameters are listed in the following:

$$
\begin{gathered}
A_{i}=\left[\begin{array}{cc}
-2 & 0 \\
0 & -0.9
\end{array}\right], \quad B_{i}=\left[\begin{array}{cc}
-1 & 0 \\
-1 & -1
\end{array}\right], \quad C_{i}=\left[\begin{array}{cc}
0.1 & 0 \\
0 & 0.1
\end{array}\right], \\
i \in S=\{1\}, \\
\alpha=0.05, \quad \beta=0.1, \quad \gamma=0, \\
d_{1}=0, \quad d_{2}=\tau, \quad \mu=\tau_{d} .
\end{gathered}
$$

For given $\varepsilon$ and $\mu$, choose $d_{m}=d_{2} / 2$ and utilize Theorem 10, and the maximum upper bound of $d_{2}$, which satisfies the LMIs in (22), (23), and (24), can be obtained by solving a quasi-convex optimization problem. The results are presented in Tables 3,4 , and 5 .

From Tables 3, 4, and 5, we consider $\mu=0, \mu=0.5$, and $\mu=0.9$ and obtain the maximum upper bound of delay $d_{2}=1.5167, d_{2}=1.0643$, and $d_{2}=0.713$, respectively, in this paper by setting $\widehat{\varepsilon}=0.1$, while the maximum upper bound of delay $d_{2}=1.2999, d_{2}=0.9442$, and $d_{2}=0.5471$, respectively, for [42], and the maximum upper bound of delay $d_{2}=1.4008, d_{2}=1.0120, d_{2}=0.6438$, respectively, for [10]. The results are also given by setting $\widehat{\varepsilon}=0.3, \widehat{\varepsilon}=0.5$, $\widehat{\varepsilon}=0.7$, and $\widehat{\varepsilon}=0.9$, and it is found that the maximum upper bound of delay in this paper is larger than those in $[10,42]$. So, it also can be seen that Theorem 10 in this paper yields less conservative results than existing results in $[10,42]$.

4.2. Systems with Partially Unknown Transition Rates. In this subsection, numerical systems with partly unknown transition rates are given to show the effectiveness of the approaches presented in this paper. 
TABLE 5: Maximum upper bound of $d_{2}$ with different $\varepsilon$ and parameter $\mu=0.9$.

\begin{tabular}{lccccrr}
\hline Methods & $\widehat{\varepsilon}$ & 0.1 & 0.3 & 0.5 & 0.7 & 0.9 \\
\hline Chen et al. [42] & $d_{2}$ & 0.5471 & 0.5015 & 0.4650 & 0.4350 & 0.4089 \\
Qiu and Cui [10] & $d_{2}$ & 0.6438 & 0.5789 & 0.5214 & 0.4954 & 0.4231 \\
Theorem 10 with $\varepsilon=2 \widehat{\varepsilon}$ & $d_{2}$ & 0.7136 & 0.6344 & 0.5987 & 0.5473 & 0.4963 \\
\hline
\end{tabular}

TABLE 6: Maximum upper bound of $d_{2}$ with $\Pi_{1}$ and different parameter $\mu$.

\begin{tabular}{lccccccccc}
\hline Methods & $\mu$ & 0.18 & 0.19 & 0.20 & 0.21 & 0.22 & 0.23 & 0.24 & 0.25 \\
\hline Xiong et al. [43] & $d_{2}$ & 0.4489 & 0.3317 & 0.2242 & 0.1814 & 0.1486 & 0.1220 & 0.0983 & 0.0781 \\
Corollary 19 & $d_{2}$ & 0.4763 & 0.3652 & 0.2573 & 0.2154 & 0.1891 & 0.1466 & 0.1134 & 0.0946 \\
\hline
\end{tabular}

Example 4. Regard Example 1 again, and we consider the practical system of PEEC model with partially unknown transition rates $\Pi=\left[\begin{array}{ll}? & ? \\ 2 & -2\end{array}\right]$ and nonlinear perturbations

$$
\begin{aligned}
\left\|f_{1}(x(t), t)\right\| & \leq 0.1\|x(t)\|, \\
\left\|f_{2}(x(t-d(t)), t)\right\| & \leq 0.2\|x(t-d(t))\|, \\
\left\|f_{3}(\dot{x}(t-\tau(t)), t)\right\| & \leq 0.1\|\dot{x}(t-\tau(t))\| .
\end{aligned}
$$

Following the same procedure in Example 1, we solve the LMIs (22), (75), and (93) in Theorem 22 and obtain the feasible solutions to guarantee the exponential stability of the uncertain neutral delay Markovian jump system with nonlinear perturbations and partially unknown transition rates.

Example 5. Consider the nominal system (9) with four operation modes $S=\{1,2,3,4\}, \alpha=\beta=\gamma=0$, and the following parameters:

$$
\begin{aligned}
& A_{1}=\left[\begin{array}{cc}
-1.15 & -0.75 \\
1.50 & -1.50
\end{array}\right], \quad B_{1}=\left[\begin{array}{cc}
-1.20 & 0.12 \\
0.24 & -0.25
\end{array}\right], \\
& C_{1}=\left[\begin{array}{cc}
-0.15 & -0.06 \\
0.50 & -0.50
\end{array}\right] \text {, } \\
& A_{2}=\left[\begin{array}{cc}
-2.15 & -0.49 \\
1.50 & -2.10
\end{array}\right], \quad B_{2}=\left[\begin{array}{cc}
-1.45 & -0.96 \\
0.47 & -1.57
\end{array}\right] \text {, } \\
& C_{2}=\left[\begin{array}{ll}
0.25 & -0.35 \\
0.05 & -0.65
\end{array}\right] \text {, } \\
& A_{3}=\left[\begin{array}{cc}
-1.30 & -0.15 \\
1.50 & -1.80
\end{array}\right], \quad B_{3}=\left[\begin{array}{cc}
0.58 & -0.68 \\
-0.13 & 0.96
\end{array}\right] \text {, } \\
& C_{3}=\left[\begin{array}{cc}
0.33 & 0.07 \\
0.19 & -0.36
\end{array}\right] \text {, } \\
& A_{4}=\left[\begin{array}{cc}
-1.90 & -0.34 \\
1.50 & -1.65
\end{array}\right], \quad B_{4}=\left[\begin{array}{cc}
-0.67 & -1.50 \\
1.39 & 1.23
\end{array}\right] \text {, } \\
& C_{4}=\left[\begin{array}{cc}
-0.23 & 0.16 \\
0.02 & -0.57
\end{array}\right] \text {. }
\end{aligned}
$$

The partially unknown transition rate matrix is considered as the following two cases:

$$
\begin{aligned}
\Pi_{1} & =\left[\begin{array}{cccc}
-1.3 & 0.2 & ? & ? \\
? & ? & 0.3 & 0.3 \\
0.6 & ? & -1.5 & ? \\
0.4 & ? & ? & ?
\end{array}\right], \\
\Pi_{2} & =\left[\begin{array}{cccc}
-1.3 & 0.2 & 0.4 & 0.7 \\
? & ? & 0.3 & 0.3 \\
0.6 & ? & -1.5 & ? \\
0.4 & ? & ? & ?
\end{array}\right] .
\end{aligned}
$$

For given $\varepsilon=0$ and $d(t)=\tau(t)$, we have $d_{1}=0, d_{2}=\tau$, and $\mu=\tau_{d}$. Set $d_{m}=d_{2} / 2$ and employ Corollary 19 , and the maximum upper bound of the time delay $d_{2}$, which satisfies LMIs (22), (75), and (84), can be calculated by solving a quasi-convex optimization problem. This neutral Markovian jump system with partially unknown transition rates was also considered in reference [43]. The results on the maximum upper bound of $d_{2}$ are compared in Tables 6 and 7 .

From Tables 6 and 7, we consider the previous system with $\Pi_{1}, \Pi_{2}$ and obtain the maximum upper bound of delay $d_{2}=0.4763, d_{2}=12.4382$, respectively, in this paper by setting $\mu=0.18$, while the maximum upper bound of delay $d_{2}=0.4489, d_{2}=12.0750$, respectively for [43]. The results are also given by setting $\mu=0.19, \mu=0.20, \mu=0.21, \mu=$ $0.22, \mu=0.23, \mu=0.24$, and $\mu=0.25$, and it is found that the maximum upper bound of delay in this paper is larger than that of [43]. So, it can be seen that our proposed method is less conservative than the result in [43]. Besides, we know that the maximum upper bound of delay to guarantee stability is dependent on transition rates knowledge.

\section{Conclusions}

This paper addresses the exponential stability for neutral Markovian jumping systems with interval time-varying delays, nonlinear perturbations, and partially unknown transition rates. According to the Lyapunov theory, a novel augmented Lyapunov-Krasovskii functional, which contains some triple-integral terms and sufficiently takes advantage of the delay bound, is constructed by dividing the time-varying delay interval into two subintervals. Then, less conservative 
TABle 7: Maximum upper bound of $d_{2}$ with $\Pi_{2}$ and different parameter $\mu$.

\begin{tabular}{lccccccccc}
\hline Methods & $\mu$ & 0.18 & 0.19 & 0.20 & 0.21 & 0.22 & 0.23 & 0.24 & 0.25 \\
\hline Xiong et al. [43] & $d_{2}$ & 12.0750 & 0.9924 & 0.5147 & 0.3749 & 0.2742 & 0.2139 & 0.1737 & 0.1433 \\
Corollary 19 & $d_{2}$ & 12.4382 & 1.2574 & 0.6253 & 0.4026 & 0.2997 & 0.2475 & 0.2015 & 0.1789 \\
\hline
\end{tabular}

delay-range-dependent and rate-dependent exponential stability criteria are obtained by the reciprocally convex lemma and free weighting matrices, both of which can be used to reduce the conservativeness. Furthermore, these theoretical results are successfully verified through some numerical examples.

\section{Acknowledgments}

This work was supported in part by the National Key Scientific Research Project (61233003), the National Natural Science Foundation of China (60935001, 61174061, 61074033, and 60934006), the Doctoral Fund of Ministry of Education of China (20093402110019), Anhui Provincial Natural Science Foundation (11040606M143), the Fundamental Research Funds for the Central Universities, and the Program for New Century Excellent Talents in University.

\section{References}

[1] V. B. Kolmanovskii and A. Myshkis, Applied Theory of Functional Differential Equations, Kluwer Academic, Dodrecht, The Netherlands, 1992.

[2] S. I. Niculescu, Delay Effects on Stability: A Robust Control Approach, Springer, Berlin, Germany, 2001.

[3] P. L. Liu, "Further results on the exponential stability criteria for time delay singular systems with delay-dependence," International Journal of Innovative Computing, Information and Control, vol. 8, no. 6, pp. 4015-4024, 2012.

[4] W. A. Zhang, L. Yu, and Sh. Yin, "A switched system approach to $H_{\infty}$ control of networked control systems with time-varying delays," Journal of the Franklin Institute, vol. 348, no. 8, pp. 165178, 2011.

[5] C. H. Lien and J. D. Chen, "Discrete-delay-independent and discrete-delay-dependent criteria for a class of neutral systems," Journal of Dynamic Systems, Measurement and Control, vol. 125, no. 1, pp. 33-41, 2003.

[6] M. N. A. Parlakçı, "Delay-dependent robust stability criteria for uncertain neutral systems with mixed time-varying discrete and neutral delays," Asian Journal of Control, vol. 9, no. 4, pp. 411421, 2007.

[7] O. M. Kwon and J. H. Park, "Delay-range-dependent stabilization of uncertain dynamic systems with interval time-varying delays," Applied Mathematics and Computation, vol. 208, no. 1, pp. 58-68, 2009.

[8] S. Y. Xu and J. Lam, "A survey of linear matrix inequality techniques in stability analysis of delay systems," International Journal of Systems Science, vol. 39, no. 12, pp. 1095-1113, 2008.

[9] C. C. Shen and S. M. Zhong, "New delay-dependent robust stability criterion for uncertain neutral systems with timevarying delay and nonlinear uncertainties," Chaos, Solitons and Fractals, vol. 40, no. 5, pp. 2277-2285, 2009.
[10] F. Qiu and B. T. Cui, "Improved exponential stability criteria for uncertain neutral system with nonlinear parameter perturbations," International Journal of Automation and Computing, vol. 7, no. 4, pp. 413-418, 2010.

[11] H. R. Karimi, M. Zapateiro, and N. Luo, "Stability analysis and control synthesis of neutral systems with time-varying delays and nonlinear uncertainties," Chaos, Solitons and Fractals, vol. 42, no. 1, pp. 595-603, 2009.

[12] J. H. Park, "Novel robust stability criterion for a class of neutral systems with mixed delays and nonlinear perturbations," Applied Mathematics and Computation, vol. 161, no. 2, pp. 413421, 2005.

[13] J. H. Zhang, P. Shi, and J. Q. Qiu, "Robust stability criteria for uncertain neutral system with time delay and nonlinear uncertainties," Chaos, Solitons \& Fractals, vol. 38, no. 1, pp. 160$167,2008$.

[14] Y. He, Q.-G. Wang, L. Xie, and C. Lin, "Further improvement of free-weighting matrices technique for systems with timevarying delay," IEEE Transactions on Automatic Control, vol. 52, no. 2, pp. 293-299, 2007.

[15] A. Tanikawa, "On new smoothing algorithms for discretetime linear stochastic systems with unknown disturbances," International Journal of Innovative Computing, Information and Control, vol. 4, no. 1, pp. 15-24, 2008.

[16] B. Chen, H. Li, P. Shi, C. Lin, and Q. Zhou, "Delay-dependent stability analysis and controller synthesis for Markovian jump systems with state and input delays," Information Sciences, vol. 179, no. 16, pp. 2851-2860, 2009.

[17] G. Zhang, C. Han, Y. Guan, and L. Wu, "Exponential stability analysis and stabilization of discrete-time nonlinear switched systems with time delays," International Journal of Innovative Computing, Information and Control A, vol. 8, no. 3, pp. 19731986, 2012.

[18] J. L. Liu, Z. Gu, and S. Hu, " $H_{\infty}$ filtering for Markovian jump systems with time-varying delays," International Journal of Innovative Computing, Information and Control, vol. 7, no. 3, pp. 1299-1310, 2011.

[19] J. L. Xiong and J. Lam, "Stabilization of discrete-time Markovian jump linear systems via time-delayed controllers," Automatica, vol. 42, no. 5, pp. 747-753, 2006.

[20] J. Wu, T. Chen, and L. Wang, "Delay-dependent robust stability and $H_{\infty}$ control for jump linear systems with delays," Systems \& Control Letters, vol. 55, no. 11, pp. 939-948, 2006.

[21] P. Shi, Y. Xia, G. P. Liu, and D. Rees, "On designing of slidingmode control for stochastic jump systems," IEEE Transactions on Automatic Control, vol. 51, no. 1, pp. 97-103, 2006.

[22] S. Xu, J. Lam, and X. Mao, "Delay-dependent $H_{\infty}$ control and filtering for uncertain Markovian jump systems with timevarying delays," IEEE Transactions on Circuits and Systems, vol. 54, no. 9, pp. 2070-2077, 2007.

[23] L. L. Xiong, X. B. Zhou, J. Qiu, and J. Lei, "Stability analysis for Markovian jump neutral systems with mixed delays and partially known transition rates," Abstract and Applied Analysis, vol. 2012, Article ID 450168, 22 pages, 2012. 
[24] L. Zhang and J. Lam, "Necessary and sufficient conditions for analysis and synthesis of Markov jump linear systems with incomplete transition descriptions," IEEE Transactions on Automatic Control, vol. 55, no. 7, pp. 1695-1701, 2010.

[25] Q. Ma, S. Xu, and Y. Zou, "Stability and synchronization for Markovian jump neural networks with partly unknown transition probabilities," Neurocomputing, vol. 74, no. 17, pp. 3404-3411, 2011.

[26] X. Luan, F. Liu, and P. Shi, "Finite-time filtering for non-linear stochastic systems with partially known transition jump rates," IET Control Theory and Applications, vol. 4, no. 5, pp. 735-745, 2010.

[27] Y. Zhang, Y. He, M. Wu, and J. Zhang, "Stabilization for Markovian jump systems with partial information on transition probability based on free-connection weighting matrices," Automatica, vol. 47, no. 1, pp. 79-84, 2011.

[28] J. K. Hale and S. M. V. Lunel, Introduction to FunctionalDifferential Equations, Applied Mathematical Sciences, Springer, New York, NY, USA, 1993.

[29] X. M. Sun, J. Zhao, and D. J. Hill, "Stability and $L_{2}$-gain analysis for switched delay systems: a delay-dependent method," Automatica, vol. 42, no. 10, pp. 1769-1774, 2006.

[30] X. Mao, "Stability of stochastic differential equations with Markovian switching," Stochastic Processes and their Applications, vol. 79, no. 1, pp. 45-67, 1999.

[31] H. B. Ji, Algebra Foundation of Control Theory, University of Science and Technology of China Press, Hefei, China, 2008.

[32] Y. G. Sun, L. Wang, and G. Xie, "Exponential stability of switched systems with interval time-varying delay," IET Control Theory \& Applications, vol. 3, no. 8, pp. 1033-1040, 2009.

[33] J. Sun, G. P. Liu, and J. Chen, "Delay-dependent stability and stabilization of neutral time-delay systems," International Journal of Robust and Nonlinear Control, vol. 19, no. 12, pp. 13641375, 2009.

[34] P. Park, J. W. Ko, and C. Jeong, "Reciprocally convex approach to stability of systems with time-varying delays," Automatica, vol. 47, no. 1, pp. 235-238, 2011.

[35] L. Xie, "Output feedback $H_{\infty}$ control of systems with parameter uncertainty," International Journal of Control, vol. 63, no. 4, pp. 741-750, 1996.

[36] D. Wang, W. Wang, and P. Shi, "Exponential $H_{1}$ filtering for switched linear systems with interval time-varying delay," International Journal of Robust and Nonlinear Control, vol. 19, no. 5, pp. 532-551, 2009.

[37] D. Wang, W. Wang, P. Shi, and X.-M. Sun, "Controller failure analysis for systems with interval time-varying delay: a switched method," Circuits, Systems, and Signal Processing, vol. 28, no. 3, pp. 389-407, 2009.

[38] S. He and F. Liu, "Exponential stability for uncertain neutral systems with Markov jumps," Journal of Control Theory and Applications, vol. 7, no. 1, pp. 35-40, 2009.

[39] S. Mondie and V. L. Kharitonov, "Exponential estimates for retarded time-delay systems: An LMI approach," IEEE Transactions on Automatic Control, vol. 50, no. 2, pp. 268-273, 2005.

[40] P. L. Liu, "Exponential stability for linear time-delay systems with delay dependence," Journal of the Franklin Institute, vol. 340, no. 6-7, pp. 481-488, 2003.

[41] S. Y. Xu, J. Lam, and M. Y. Zhong, "New exponential estimates for time-delay systems," IEEE Transactions on Automatic Control, vol. 51, no. 9, pp. 1501-1506, 2006.
[42] Y. Chen, A. Xue, R. Lu, and S. Zhou, "On robustly exponential stability of uncertain neutral systems with time-varying delays and nonlinear perturbations," Nonlinear Analysis. Theory, Methods \& Applications, vol. 68, no. 8, pp. 2464-2470, 2008.

[43] L. L. Xiong, J. B. Tian, and X. Z. Liu, "Stability analysis for neutral Markovian jump systems with partially unknown transition probabilities," Journal of the Franklin Institute, vol. 349, no. 6, pp. 2193-2214, 2012. 


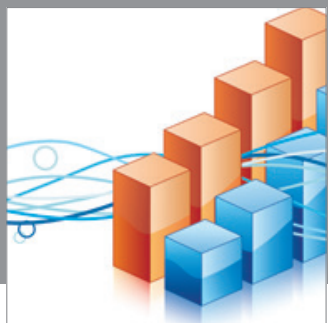

Advances in

Operations Research

mansans

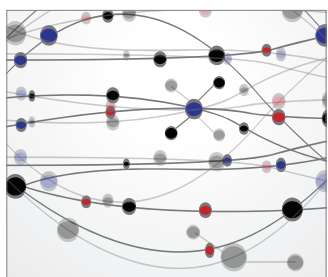

The Scientific World Journal
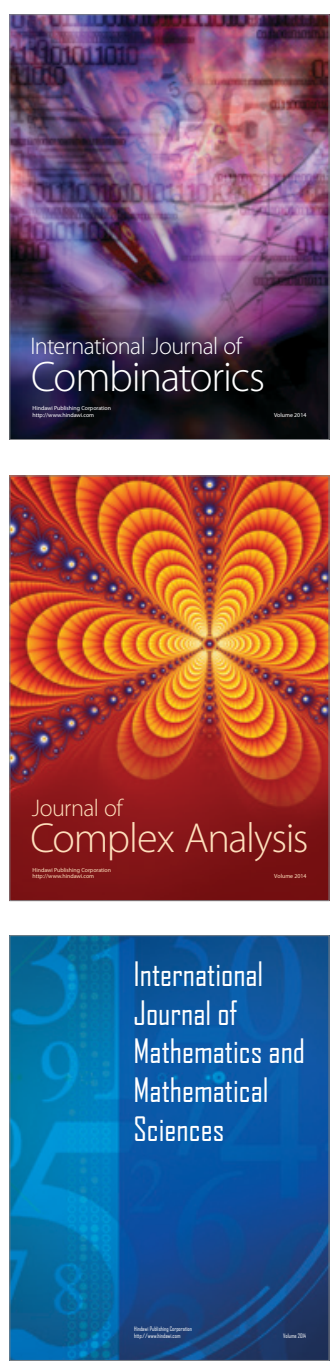
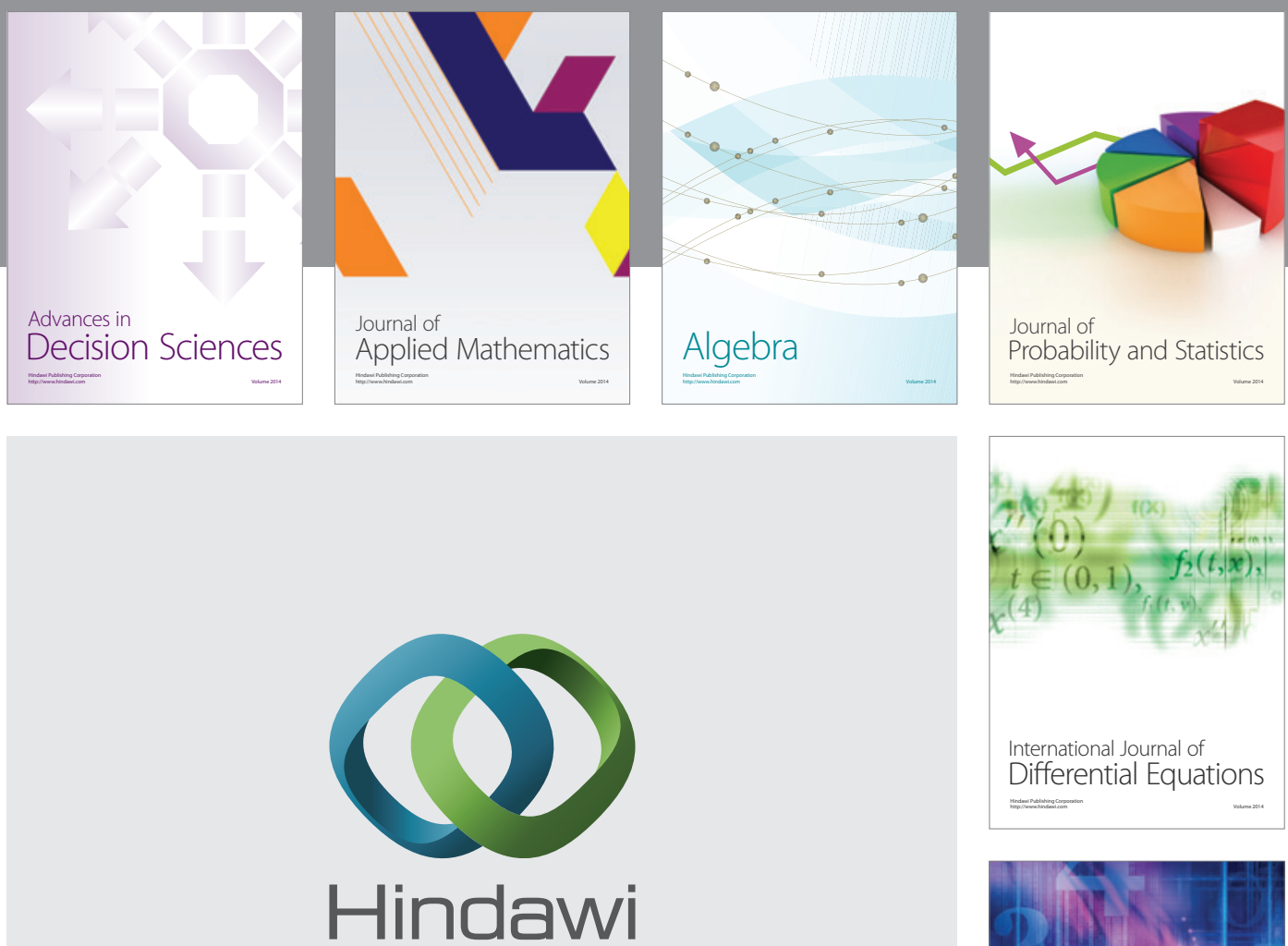

Submit your manuscripts at http://www.hindawi.com
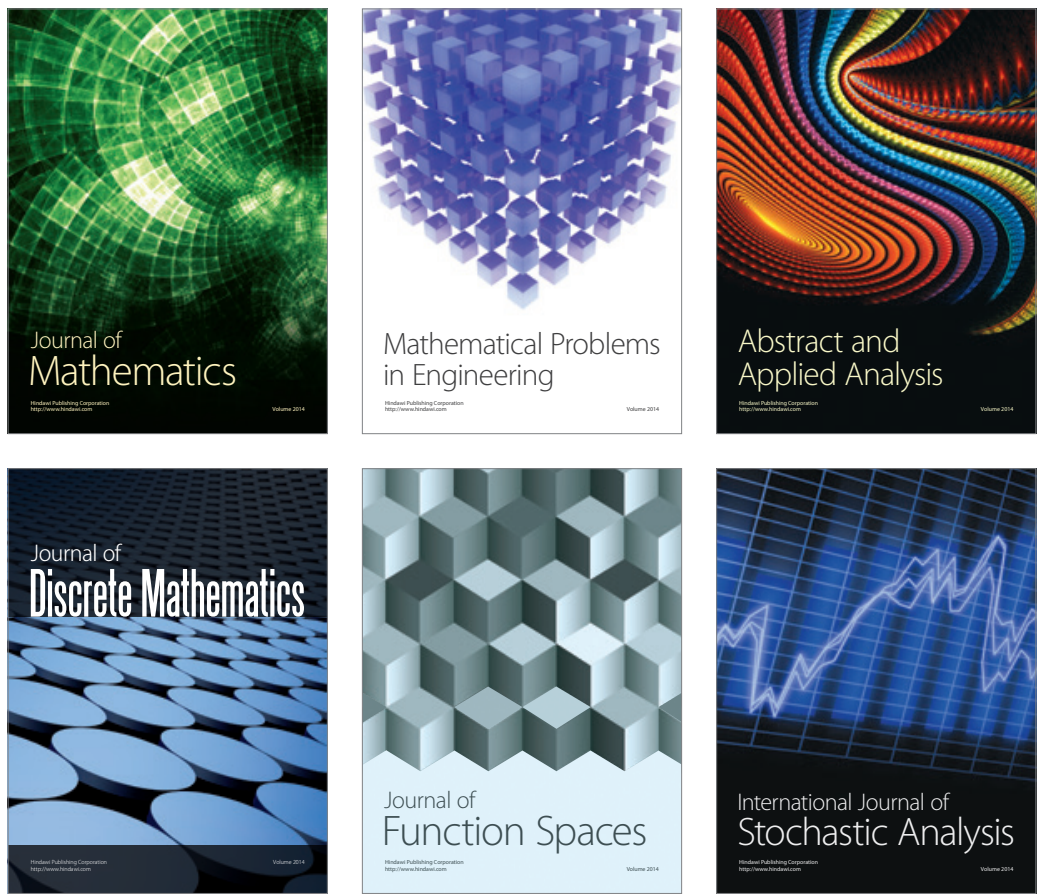

Journal of

Function Spaces

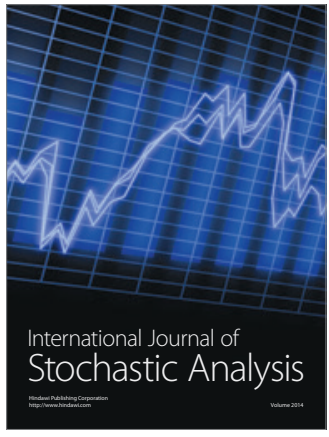

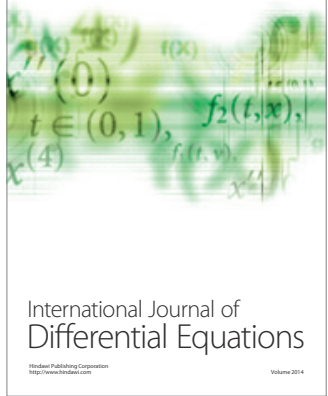
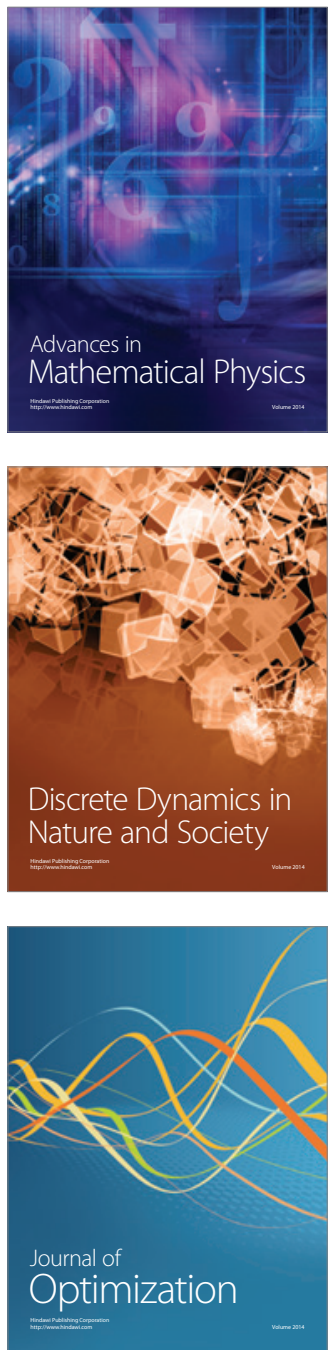